Listening for the Mars 2020 Landing Sequence with InSight

\title{
Listening for the Landing: Seismic Detections of Perseverance's arrival at Mars with InSight
}

\author{
Benjamin Fernando ${ }^{1}$, Natalia Wójcicka ${ }^{2}$, Marouchka Froment $^{3,4}$, Ross \\ Maguire $^{5,6}$, Simon C. Stähler ${ }^{7}$, Lucie Rolland ${ }^{8}$, Gareth S. Collins ${ }^{2}$, Ozgur \\ Karatekin $^{9}$, Carene Larmat ${ }^{3}$, Eleanor K. Sansom ${ }^{10}$, Nicholas A. Teanby ${ }^{11}$, \\ Aymeric Spiga ${ }^{12,13}$, Foivos Karakostas ${ }^{5}$, Kuangdai Leng ${ }^{14}$, Tarje \\ Nissen-Meyer $^{1}$, Taichi Kawamura ${ }^{4}$, Domenico Giardini ${ }^{7}$, Philippe Lognonné ${ }^{4}$, \\ Bruce Banerdt ${ }^{15}$, Ingrid J. Daubar ${ }^{16}$
}

\footnotetext{
${ }^{1}$ Department of Earth Sciences, University of Oxford, South Parks Road, Oxford, OX1 4AR, UK ${ }^{2}$ Department of Earth Science and Engineering, Imperial College, London, SW7 2AZ, UK ${ }^{3}$ Earth and Environmental Sciences Division, Los Alamos National Laboratory, Los Alamos, NM, USA ${ }^{4}$ Université de Paris, Institut de Physique du Globe de Paris, CNRS, Paris, France ${ }^{5}$ Department of Geology, University of Maryland, College Park, MD, USA

${ }^{6}$ Department of Computational Mathematics, Science, and Engineering, Michigan State University, East Lansing, MI, USA

${ }^{7}$ Department of Earth Sciences, ETH Zurich, Sonneggstrasse 5, 8092 Zürich, Switzerland

${ }^{8}$ Université Côte d'Azur, Observatoire de la Côte d'Azur, CNRS, IRD, Géoazur, France ${ }^{9}$ Royal Observatory of Belgium, Belgium ${ }^{10}$ Space Science and Technology Centre, Curtin University, Australia

${ }^{11}$ School of Earth Sciences, University of Bristol, Wills Memorial Building, Queens Road, Bristol BS8 1RJ, UK

${ }^{12}$ Laboratoire de Météorologie Dynamique / Institut Pierre-Simon Laplace (LMD/IPSL), Sorbonne Université, Centre National de la Recherche Scientifique (CNRS), École Polytechnique, École Normale Supérieure (ENS), Campus Pierre et Marie Curie BC99, 4 place Jussieu 75005 Paris, France ${ }^{13}$ Institut Universitaire de France (IUF), 1 rue Descartes, 75005 Paris, France ${ }^{14}$ Scientific Computing Department, Rutherford Appleton Laboratory, Harwell, UK

${ }^{15}$ Jet Propulsion Laboratory, California Institute of Technology, Pasadena, CA, USA

${ }^{16}$ Earth, Environment and Planetary Sciences, Brown University, Providence, RI, USA
}

\section{Key Points:}

- The entry, descent and landing of Mars 2020 (NASA's Perseverance Rover) will act as a seismic source on Mars

- We evaluate the detectability of the acoustic (atmospheric) and elastodynamic seismic (ground) signals

- We predict the acoustic signal will not likely be detectable by InSight, but the seismic signal may be. 


\begin{abstract}
The entry, descent, and landing (EDL) sequence of NASA's Mars 2020 Perseverance rover will act as a seismic source of known temporal and spatial localization. We evaluate whether the signals produced by this event will be detectable by the InSight lander (3452 km away), comparing expected signal amplitudes to noise levels at the instrument. Modeling is undertaken to predict the propagation of the acoustic signal (purely in the atmosphere), the seismoacoustic signal (atmosphere-to-ground coupled), and the elastodynamic seismic signal (in the ground only). Our results suggest that the acoustic and seismoacoustic signals, produced by the atmospheric shockwave from the EDL, are unlikely to be detectable due to the pattern of winds in the martian atmosphere and the weak air-toground coupling, respectively. However, the elastodynamic seismic signal produced by the impact of the spacecraft's cruise balance masses on the surface may be detected by InSight. The upper and lower bounds on predicted ground velocity at InSight are $2.0 \times$ $10^{-14} \mathrm{~ms}^{-1}$ and $1.3 \times 10^{-10} \mathrm{~ms}^{-1}$. The upper value is above the noise floor at the time of landing $40 \%$ of the time on average. The large range of possible values reflects uncertainties in the current understanding of impact-generated seismic waves and their subsequent propagation and attenuation through Mars. Uncertainty in the detectability also stems from the indeterminate instrument noise level at the time of this future event. A positive detection would be of enormous value in constraining the properties of the martian atmosphere, crust, and mantle as well as in improving our understanding of impactgenerated seismic waves.
\end{abstract}

\title{
Plain Language Summary
}

When it lands on Mars, NASA's Perseverance Rover will have to slow down rapidly to achieve a safe landing. In doing this, it will produce a sonic boom and eject two large balance masses which will hit the surface at very high speed. The sonic boom and balance mass impacts will produce seismic waves which will travel away from Perseverance's landing site. Here we evaluate whether these seismic waves will be detectable by instruments on the InSight lander (3452 km away). We predict that the waves from the balance mass impacts may be detectable. If the waves are recorded by InSight, this would represent the first detection of ground motion generated by a seismic source on Mars at a known time and location. This would be of enormous value in advancing our understanding of the structure and properties of Mars' atmosphere and interior as well as in improving our understanding of impact-generated seismic waves.

\section{Introduction}

\subsection{Motivation}

NASA's Interior Exploration using Seismic Investigations, Geodesy and Heat Transport (InSight) mission landed on Mars' Elysium Planitia in November 2018, and since then has detected a number of 'marsquake' events which are thought to be geologitectonic in origin (Banerdt et al., 2020).

InSight faces a number of peculiar challenges associated with single-station seismology (Panning et al., 2015). Without independent constraints on source properties, robust seismic inversions are more challenging than they would be on Earth. Impact events (where meteoroids hit the planet's surface) offer an opportunity to overcome some of these challenges as they can be photographically constrained in location, size and timing from orbital images. In theory, this should allow a positive impact detection to be used as 'calibration' for other seismic measurements.

However, no impact events have yet been conclusively detected and identified by InSight, despite pre-landing expectations that impacts would make a significant contri- 
bution to martian seismicity (Daubar et al., 2018). A meteorite impact which formed a new $1.5 \mathrm{~m}$ impact crater only $37 \mathrm{~km}$ from InSight in 2019 was not detected (Daubar et al., 2020).

A number of possible reasons may explain the absence of impact detections thus far. These include uncertainties in the impactor flux entering Mars' atmosphere (Daubar et al., 2013) and in the seismic efficiency (the fraction of impactor kinetic energy converted into seismic energy) of ground impacts that form metre-scale craters (Wójcicka et al., 2020), as well as high ambient noise through much of the day, which makes detecting faint signals challenging. Should a seismic signal excited by an impact be detected, distinguishing it from tectonic events remains challenging due to intense scattering in the shallow crust of Mars (see van Driel et al. (2019) or Daubar et al. (2020) for further discussion).

If a seismic signal recorded by InSight could be identified as impact-generated, conclusive attribution to a particular spatial and temporal location would require identification of a new crater on the surface. Sparse orbital imaging coverage of the martian surface at the required resolution, coupled with large error bounds on event distance and azimuth estimations (e.g. Giardini et al. (2020)), make this extremely challenging. This The use of orbital imagery also excludes seismic offers no information about seismic or infrasonic signals induced by those impactors which either burn up or explode in the at-

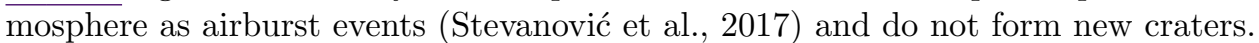

These challenges may be overcome by using as seismic sources the Entry, Descent, and Landing (EDL) sequences of objects with known entry ephemerides (meaning a priori calculated or independently constrained entry/re-entry timings and locations. On Mars, a very limited number of events with known atmospheric entry ephemerides, and therefore a priori known times and locations. The Mars 2020 mission, landing in February 2021, offers an opportunity for this possible measurement.

\subsection{Terrestrial and lunar context}

Spacecraft re-entering the atmosphere are comparatively common on Earth. These have trajectories which are often known prior to their arrival, meaning that seismic observation campaigns can be planned in advance. This has been done for a variety of spacecraft, including the Apollo command capsules (Hilton \& Henderson, 1974), the Space Shuttle (Qamar, 1995; de Groot-Hedlin et al., 2008), Hayabusa-1 (Ishihara et al., 2012), Genesis (ReVelle et al., 2005) and Stardust (ReVelle \& Edwards, 2007). In these cases, seismic and infrasonic data were used to study the entry dynamics of the spacecraft in question, for example the mechanics of energy dissipation into the atmosphere.

Naturally occurring impact events on Earth may not have trajectories which are known in advance, but their flight paths may be independently reconstructed from photographic evidence (e.g. Devillepoix et al. (2020)) or the recovery of fragments. Examples include the Carancas impact which occured in Peru in 2007 (Le Pichon et al., 2008; Tancredi et al., 2009) and the Chelyabinsk airburst in Russia in 2013 (Borovička et al., 2013). In such studies, seismic and infrasonic measurements (Tauzin et al., 2013; de Groot-Hedlin \& Hedlin, 2014) are used to study both the entry dynamics and also the properties of the meteoroids themselves, for example radii, masses, and rates of ablation.

On Earth the density of seismic stations and frequency of tectonic events means that impacts are not needed for calibration purposes. However, the Apollo Seismic Experiment did use artificial impacts for calibration on the moon (Nakamura et al., 1982). In this case, the sources were the impacts of the spent upper stages of the Saturn $\mathrm{V}$ rockets or derelict Lunar Modules with the lunar surface, which were detected 
by a network of seismometers deployed by the Apollo astronauts. These events had a known time and location of impact, enabling exact identification of travel times and ray propagation paths for the resulting seismic waves to be made.

\subsection{Extension of these methods to Mars}

On Mars, spacecraft entering the atmosphere are rare. The presence of an atmosphere complicates modelling of impact processes as compared to the lunar case (Nunn et al., 2020), and the entirely different surface and atmospheric compositions mean terrestrial analogues are not directly applicable either (Lognonné et al., 2016). Specifically, the presence of a dry, weakly cohesive surface regolith layer on Mars is expected to reduce the seismic efficiency of impacts as compared to Earth (Wójcicka et al., 2020), whilst the high $\mathrm{CO}_{2}$ concentration in the atmosphere attenuates highfrequency acoustic signals much more rapidly on Mars than on Earth (Williams, 2001; Lognonné et al., 2016; Bass \& Chambers, 2001).

The landing of NASA's Mars 2020 Rover (Perseverance) on February 18, 2021 will beis the first time that an EDL event has occurred on Mars during the lifetime of the InSight lander. This paper informs the first ever attempted EDL detection on surface of another planet. InSight's potential to detect EDL sequences has, however, proved a source of inspiration in the popular media (Away, Season 1, Episode 8, 2020).

The few that do occur are the entry, descent, and landing (EDL) sequences of human-made spacecraft. Whilst such detections have previously been achieved on Earth (de Groot-Hedlin et al., 2008), and spacecraft impact signals have been used as exemplar seismic sources on the Moon (Nunn et al., 2020)

no seismic detection of an EDL on another planet has ever occurred

Seismic signals from EDL events are of significant interest from a seismological point of view. If detected, seismic signals from EDL events they would enable us to both better constrain the seismic efficiency and impact processes for those bodies which strike the surface (as the inceming mass, velocity and angle are all known). would enable us to place substantially better constraints on the seismic efficiency of small impacts on Mars (for those parts of the EDL apparatus which strike the surface) and the generation of seismic waves by impacts.

An artificial impact also confers the advantage that the impactor mass, velocity, radius, and angle of flight with respect to the ground are all known to within a high degree of precision well in advance, and post-landing return of flight trajectory data and imaging of the resultant craters can provide further constraints (Bierhaus et al., 2013).

they A positive detection would also be of substantial benefit to planetary geophysics more generally, enabling us to calibrate the source and structural properties derived from other marsquake events which do not have a priori known source parameters. A negative detection would also be useful, enabling us to place upper bounds on these signals' amplitudes and hence to better constrain the scaling relationships used to predict the amplitudes of seismic waves from impact events.

Finally, we alse hope that the workflow developed here to evaluate the seismic detectability of EDL signals will be of use in future planetary seismology missions.

The next EDL sequence to occur on Mars will be that of NASA's Mars 2020 (Perseverance) rover on February 18, 2021, which is the focus of this paper. We aim to estimate the amplitudes of the seismic signals this will produce at InSight's location, and hence estimate their detectability. 


\subsection{The Mars 2020 EDL Sequence: parameters}

Perseverance's landing is targeted for approximately 15:15 Local True Solar Time (LTST) on February 18, 2021. This corresponds to 18:55 LTST at InSight $\left(4.50^{\circ} \mathrm{N}, 135.62^{\circ} \mathrm{E}\right)$, or roughly 20:55 UTC on Earth. The centre of the $10 \mathrm{~km}$ by $10 \mathrm{~km}$ landing ellipse is within Jezero Crater at $18.44^{\circ} \mathrm{N}, 77.50^{\circ} \mathrm{E}$ (Grant et al., 2018). At atmospheric interface $(125 \mathrm{~km}$ altitude), the spacecraft's entry mass is $3350 \mathrm{~kg}$ and the heat shield is $4.5 \mathrm{~m}$ in diameter. At this point the spacecraft's velocity is approximately $19,200 \mathrm{~km} / \mathrm{h}$, and it is accelerating.

This is a distance of $3452 \mathrm{~km}$ nearly due west from InSight. During descent the spacecraft trajectory is along an entry azimuth trajectory of approximately $100^{\circ}$ (Figs. 1 and $3 \mathrm{a}$ ), or pointing eastward (azimuth $105^{\circ}$ ) and directed almost exactly towards InSight.

Two portions of the EDL sequence are likely to produce strong seismic signals. The first is the period during which the spacecraft is generating a substantial Mach shock as it decelerates in the atmosphere, and the second is the impact of the spacecraft's two Cruise Mass Balance Devices (CMBDs) on the surface.

note that six smaller balance masses which impact at much lower velocities are not appreciable seismic sources and are not considered in this paper).

The spacecraft will generate a sonic boom during descent, from the time at which the atmosphere is dense enough for substantial compression to occur (an altitudes around $100 \mathrm{~km}$ and below), until the spacecraft's speed becomes sub-sonic, just under 3 minutes prior to touchdown. The maximum deceleration will be at around $30 \mathrm{~km}$ altitude. This sonic boom will rapidly decay into a linear acoustic wave, with some of its energy striking the surface and undergoing seismoacoustic conversion into elastodynamic seismic waves, whilst some energy remains in the atmosphere and propagates as infrasonic pressure waves.

The second part of the EDL sequence which will generate a seismic signal is the impacts of the CMBDs on the ground. The CMBDs are dense, $77 \mathrm{~kg}$ unguided tungsten blocks which are jettisoned high in the EDL sequence (around 1,450 km altitude). Due to their high ballistic coefficients, they are expected to undergo very limited deceleration before impact. Based on simulations and data from the Mars Science Laboratory/Curiosity Rover's EDL in 2012 (Bierhaus et al., 2013) and simulations of the Mars 2020 EDL, the CMBD impacts are is expected to occur at about $4000 \mathrm{~m} / \mathrm{s}$, less than $100 \mathrm{~km}$ from $\overline{\text { the }}$ spacecraft landing site, and at an impact angle of about $10^{\circ}$ elevation from the horizontal plane (Bierhaus et al., 2013).

In the case of Curiosity, the CMBDs formed several craters between 4 and $5 \mathrm{~m}$ in diameter, and the separation between CMBDs or their resulting fragments was no more than $1 \mathrm{~km}$ at impact (Bierhaus et al., 2013), implying a difference in impact time of less than 1 second between them.

It should be noted that the CMBDs are not the only parts of the EDL hardware which will experience an uncontrolled impact. The heat shield, backshell and descent stage are also expected to reach the surface intact. However, in an optimal landing scenario these are expected to be at sub-sonic speeds (less than $100 \mathrm{~ms}^{-1}$ for masses of 440, 600, and $700 \mathrm{~kg}$ respectively). Six smaller $25 \mathrm{~kg}$ balance masses are also ejected much closer to the surface, and at considerably lower speeds. As such, no other component of the EDL hardware impacting the surface is expected to produce a seismic signal of comparable magnitude to the CMBD impact.

\subsection{Aims}

There is a clear scientific case for 'listening' for Perseverance's landing using InSight's instruments. Doing this requires comprehensive modelling of the propagation 


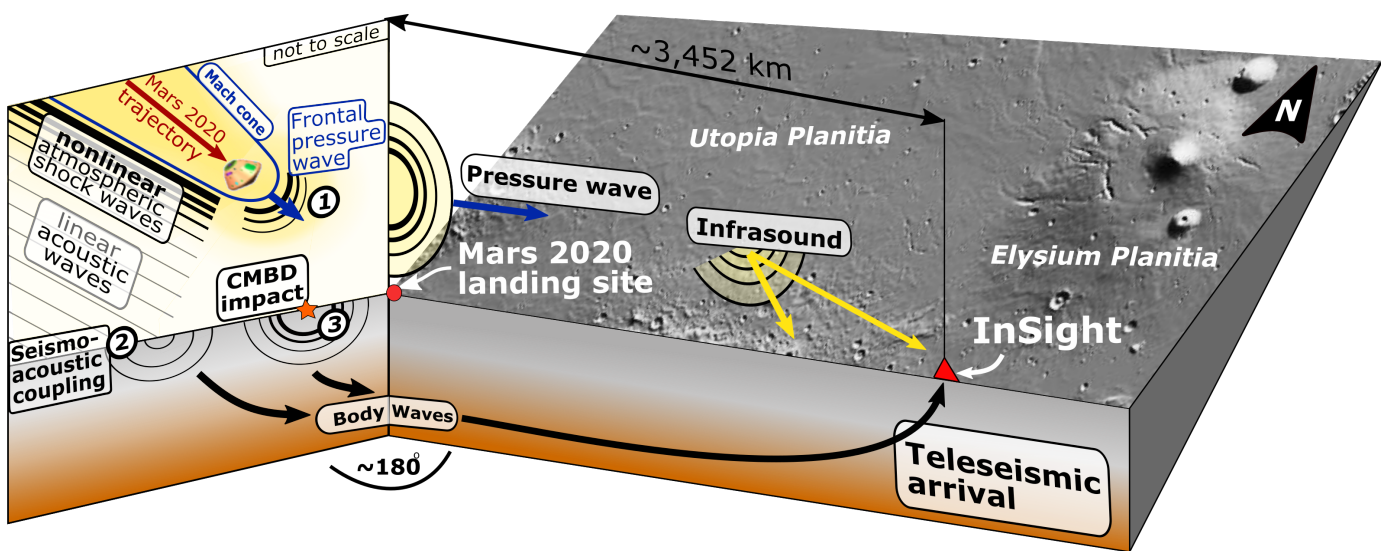

Figure 1. Schematic illustration of the seismic signals produced by the Mars 2020 EDL sequence (not to scale). Numbered features are: (1) the atmospheric acoustic signal, (2) the coupled seismoacoustic signal, and (3) the seismic signal propagating in the ground. The thickest airborne black lines represent non-linear shockwaves, decaying to weakly non-linear (thin black lines) and finally linear acoustic waves (thin gray lines). Surface waves, which on Mars do not appear to propagate at teleseismic distances, are not shown here. Black lines with single arrowheads represent body waves. The spacecraft's trajectory at entry is eastward along an azimuth of $100^{\circ}$, almost exactly pointing toward InSight, i.e. the two panels are angled toward each other at nearly $180^{\circ}$, but are shown as they are here to acknowledge remaining uncertainties in the exact entry trajectory which exist at the time of writing. Note that this figure shows all three potential sources of seismic signal, and is not intended to suggest that these all reach InSight at detectable amplitudes.

of such signals (both in the atmosphere and in the solid ground) from Perseverance's landing site to InSight, in order to estimate signal amplitudes and travel times. This paper presents this modelling work, which is being used to inform the configuration of InSight's instruments in advance of the landing.

\section{Methodology}

To assess their detectability at InSight, we consider three aspects distinct types of signals generated by Perseverance's EDL. Each of these represents wave propagation in a different medium or combination thereof: atmosphere, coupled atmosphere-ground, and ground. Corresponding to the labels in Fig. 1, these are:

1. Acoustic signal: A linear, acoustic wave propagating in the atmosphere as an infrasonic (low frequency, $<20 \mathrm{~Hz}$ ) pressure wave, generated by the decay of the sonic boom produced during descent. The modelling methodology for this portion of the signal is presented in section 2.1 , and the results in section 3.1.

2. Coupled seismoacoustic signal: A coupled air-to-ground wave, produced by the sonic boom, or its linear decay product, impinging upon the surface and creating elastodynamic body waves. On Earth, this would usually produce detectable surface waves too - however on Mars these are rapidly scattered away to non-detectable levels and hence are not depicted here. Methodology and results are in sections 2.2 and 3.2 respectively. 
3. Elastodynamic signal: An elastodynamic wave ('conventional' seismic wave) travelling in the solid part of the planet, excited by the impact of the CMBDs. Methodology and results are in sections 2.3 and 3.3 respectively.

In addition to the CMBDs, various other parts of the EDL hardware will impact the surface, including the heat shield, backshell and descent stage. However, in an optimal landing scenario these are expected to be at sub-sonic speeds (less than $100 \mathrm{~ms}^{-1}$ for masses of 440,600 , and $700 \mathrm{~kg}$ respectively) and as such will not produce seismic signals of comparable magnitude to the CMBD impact.

\subsection{Acoustic signal: Source and propagation}

The shockwave produced by the hypersonic deceleration of the spacecraft will rapidly decay through viscous frictional processes into a linear acoustic wave. The resultant acoustic (pressure) waves will propagate in the atmosphere following paths determined by the atmospheric structure. The amplitude of any potential signal at the location of InSight is determined by the decay of the signal with increasing distance; due to attenuation in the atmosphere, transmission into the ground, and geometrical spreading.

\subsubsection{Signal amplitudes}

The spacecraft is treated as a cylindrical line source, which is justified on the grounds that the opening angle of the Mach cone is small at hypersonic velocities. Solving the weak shock equations (ReVelle, 1976; Edwards, 2009; Silber et al., 2015), with additional calculations based upom Varnier et al. (2018), enables us to estimate the energy dissipated into the atmosphere by the spacecraft's entry with increasing distance from its trajectory line.

As per the weak shock theory and sonic boom formulations of the above literature sources, the overpressure decreases with increasing distance from the source as $x^{-3 / 4}$ and the source wave period increases as $x^{1 / 4}$; where $x=\frac{r}{R}$ is the distance from the line source $r$, normalised by the blast wave relaxation radius $\left(R_{0}\right) . R_{0}$ is the distance from the line source at which the overpressure approaches the ambient atmospheric pressure, and for a spherical source is approximately equal to the impactor diameter multiplied by its Mach number.

The calculations account for the gradual nature of the transition between a weakly non-linear and fully linear propagation regime; but do not include attenuation (this is discussed further in Sec 4).

As discussed further below, acoustic energy in the atmosphere may be trapped in waveguide layers, which enable low-attenuation long-distance propagation of atmospheric waves. The decay in amplitude with increasing distance from the source $r$ for waves propagating within a waveguide is poorly constrained, with both terrestrial and martian predictions falling into a range between $r^{-1}$ and $r^{-1.5}$ (Martire et al., 2020; Ens et al., 2012). If acoustic waves are trapped within a waveguide, these scaling laws enable us to predict their amplitude far from the source.

\subsubsection{Wave trajectories}

The acoustic wave trajectories are modelled using the WASP (Windy Atmospheric Sonic Propagation) software (Dessa et al., 2005). The propagation medium is a stratified atmosphere parameterised using a 1D effective sound speed (Garcia et al., 2017). This effective sound speed accounts for the presence of directional waveguides in the martian atmosphere at certain times of day, caused by wind. Wind effects are therefore fully

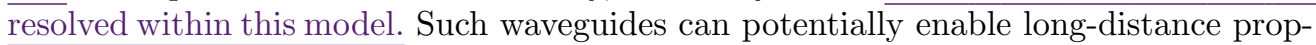


agation of an infrasonic signal in the direction of the wind. However, atmospheric waveguides are comparatively rarer than on Earth and exist only in the presence of winds, unlike on Earth where temperature inversions may create waveguides without wind (Garcia et al. (2017), Martire et al. (2020)).

The adiabatic sound speed and horizontal wind speed along the great circle propagation path from Mars 2020 to InSight are computed from the Mars Climate Database (Millour et al., 2015), accounting for the variation in local time as the signal propagates at the time and location of Perseverance's landing remove, early evening at InSight). Supplement Fig. S32 shows the variation in effective sound speed with azimuthtoward and away from InSight, highlighting that the effects of the wind are highly directional.

The atmospheric dust content, which significantly influences globartian wind and weather patterns through changes in opacity, is chosen as an average for the solar longitude $\mathrm{L}_{s}=5^{\circ}$ (northern spring) season, in which dust storms are rare (Montabone et al., 2015).

Weather perturbations may cause second-order changes in the atmospheric conditions (Banfield et al., 2020), but would not change the overall dynamics of acoustic wave propagation considered here. Regardless, in general the martian atmosphere in the equatorial regions in the northern spring is typically predictable in its meteorology (Spiga et al., 2018).

Infrasonic signals, if at detectable levels, could be recorded directly by InSight's APSS (Auxiliary Payload Sensor Suite) instrument (Banfield et al., 2019); or indirectly by InSight's SEIS (Seismic Experiment for Interior Structure) instrument (Lognonné et al., 2019). The former records the actual atmospheric pressure perturbation, whilst the latter detects the compliance-induced displacement of the ground by atmospheric overpressure or underpressure.

\subsection{Coupled seismoacoustic signal}

The impactincidence of the linear acoustic waves from the atmosphere (the products of the decaying shockwave) hittingupon the surface will excite elastodynamic (i.e. body and surface) waves in the solid ground. The other crucial-The dominant parameters in determining the amplitude of the elastodynamic waves in the solid ground isare the air-to-ground coupling factor (which is a transmission coefficient), and the value of the overpressure at the surface.

Using the method of Sorrells et al. (1971), we estimate the air-to-ground coupling factor by modelling the intersection of a planar acoustic wave with a regolith-like target material, with a density of $1270 \mathrm{kgm}^{-3}$, a P-wave velocity of $340 \mathrm{~ms}^{-1}$, and Swave velocity of $200 \mathrm{~ms}^{-1}$. The effective sound speed is derived from the Mars Climate Database (see Figure 2).

Full details of the method are described in the Supplement (Text S1), however this value is found to be $4 \times 10^{-6} \mathrm{~ms}^{-1} \mathrm{~Pa}^{-1}$. It is thus possible to proced to predicting amplitudes at InSight.

We obtain a value for the air-to-ground coupling factor of $4 \times 10^{-6} \mathrm{~ms}^{-1} \mathrm{~Pa}^{-1}$, which is of the same order of magnitude as on Earth. This value is also similar to values obtained by Garcia et al. (2017); Martire et al. (2020).

The atmospheric overpressure at ground level is modelled as described in Sec. 2.1.1, and multiplied by the derived air-to-ground coupling factor to calculate the energy transmitted into the solid ground.

After the wave has coupled into the ground, its amplitude decay upon propagating through a 1D seismic Mars model is calculated using Instaseis (van Driel et al., 2015). 

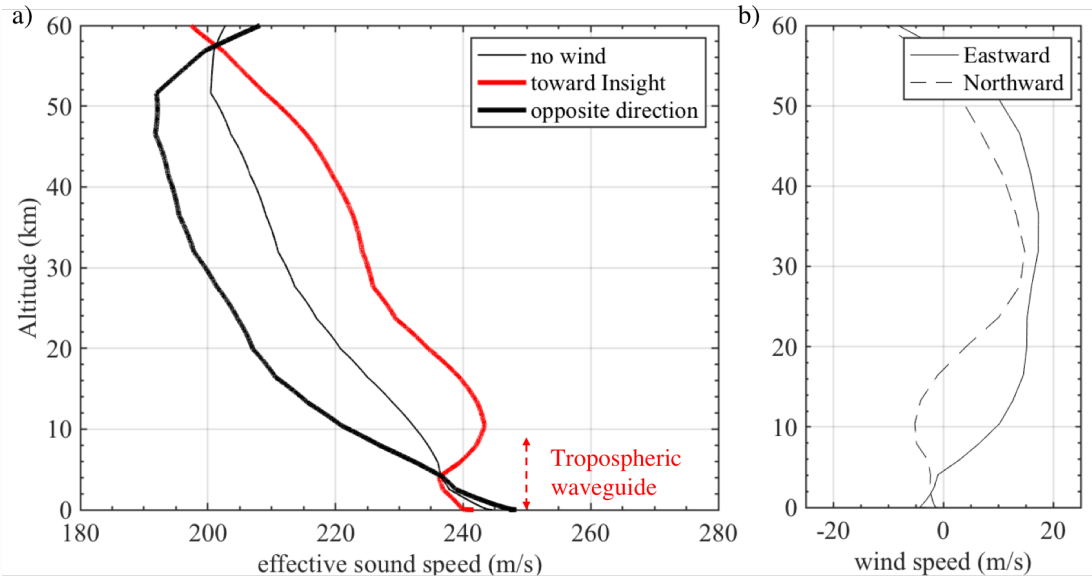

Figure 2. [Added figure.]The atmospheric model used in simulations of acoustic wave propagation, plotted at the Perseverance landing site and representative of the atmosphere state along the great circle path toward InSight. The left panel shows the effective sound speed as a function of altitude to highlight that these effects are highly directional; whilst the right panel shows the horizontal wind strength. In the absence of wind the sound speed in the atmosphere monotonically decreases between the surface and $\sim 50 \mathrm{~km}$. This is not favourable for the long-range propagation of acoustic waves, but the eastward (zonal) wind modifies this to yield an 'effective' sound speed. This creates a tenuous tropospheric waveguide at the bottom of the atmosphere in the direction toward InSight (which is on an azimuth $104^{\circ}$ from North from the landing site). The height of this waveguide is marked with a red dashed arrow. All parameters are derived from the Mars Climate Database (Millour et al., 2015). 


\subsubsection{Body Waves}

We focus on The seismoacoustically coupled direct-arrival body waves (observed on Earth from EDL impacts by Edwards et al. (2007)), which travel through the deeper parts of the crust and mantle, where less scattering attenuation is expected than in the shallow crust.

We focus on the prediction of P-wave amplitudes from seismoacoustic coupling, as these are expected to be the strongest of the direct-arrival body waves generated by atmospheric overpressure at the surface

Multiplying the value obtained for the overpressure at ground level by the air-toground coupling factor (section 2.2) gives an upper bound enfor the velocity amplitude of the $\mathrm{P}$-wave at the landing site.

The decay of this amplitude with distance to InSight's position can then be calculated using either waveform modeling or scaling laws (these are discussed below). The S-wave amplitude from the coupled seismoacoustic signal is expected to be much smaller, as the vertical incidence of the atmospheric acoustic wave produces much stronger pressure perturbations than shear perturbations in the solid ground.

The resulting body waves propagating in the solid ground large enough in amplitude, be detected by SEIS InSight's SEIS (Seismic Experiment for Interior Structure) instrument (Lognonné et al., 2019).

\subsubsection{Surface waves}

Modeling of the excitation of atmospherically induced surface waves is discussed in detail by Lognonné et al. (2016) and Karakostas et al. (2018). However, the combination of a small transmission coefficient and strong seismic scattering in the portions of the crust where the surface waves propagate (Banerdt et al., 2020) means that the surface wave signal is extremely unlikely to be detected at InSight and we do not consider itthem further in this paper. If this procedure is applied to other planetary seismology settings where surface waves are expected, they should be considered as well as they may be greater in amplitude than the P-wave. Extending the use of Instaseis to achieve this is simple.

\subsection{Elastodynamic seismic signal}

The impact of the CMBDs at the Perseverance landing site will excite both surface and body waves. As was the case for the coupled seismocacoustic signal discussed in Sec 2.2, the surface wave phases are expected to be scattered away before they reach InSight. We therefore focus on the signals which we expect to have the largest amplitude, that is, the direct-arrival P-wave. amplitudes at InSight of the seismic waves produced by the CMBD impacts at InSight, and hence to evaluate their potential detectability by SEIS.

The dynamics calculations for the spacecraft's re-entry prior to CMBD jettison, which confirm the CMBD impact parameters based on data from the Mars Science Laboratory in 2020, (Karlgaard et al., 2014)-are also-discussed in the Supplement.

The entry trajectories of Perseverance and its CMBDs are obtained through aerodynamic simulations whose inputs (i.e. the initial trajectory state, vehicle mass, aerodynamic coefficients) are considered identical to those used by the Mars Science Laboratory Curiosity (Karlgaard et al., 2014), on account of the nearly identical EDL apparatus. The aerodynamic coefficients and vehicle mass are assumed to be constant 
along the trajectory. An ellipsoid gravity model is used, and atmospheric conditions are extracted from the Mars Climate Database (MCD) climatology scenario at the predicted landing location and time.

Two approaches are taken to estimate the peak P-wave amplitudes at InSight produced by the CMBD impacts, and hence to evaluate their detectability by SEIS. The first (Sec 2.3.1) makes use of empirical amplitude scaling relationships to directly estimate the P-wave amplitude at InSight's position. The second makes use of wave propagation modelling, with the choice of source magnitude informed either by scalingbased moment estimates (Sec 2.3.2 A) or by shock physics simulations of the CMBD impact (Sec 2.3.2 B). These approaches are complementary.

\subsubsection{Method 1: Empirical amplitude scaling relationships}

The first approach uses the empirical scaling relations of Teanby (2015) and Wójcicka et al. (2020) to estimate the peak P-wave amplitudes at InSight's location. The amplitudes of the S-wave are significantly harder to estimate (and are not predictable from the published scaling relationships discussed below), but are likely to be of the same order of magnitude as the P-waves.

The empirical scaling relationships are based on the measured P-wave amplitudes as a function of distance from artificial lunar (Latham, Ewing, et al., 1970) and terrestrial missile impact experiments (Latham, McDonald, \& Moore, 1970), which follow a $r^{-1.6}$ relationship (Teanby, 2015). The approaches differ in how these relationships are rescaled to the CMBD impacts on Mars based on impactor properties. Full details of the differences between these approaches are included in the Supplementary Information. The Teanby (2015) approach scales the empirically derived P-wave amplitude with the square root of the impactor's kinetic energy; whilst the Wójcicka et al. (2020) approaches use a scaling based on impactor momentum, either total or vertical. The estimated impact energy, total momentum and vertical-component momentum of the CMBD impact are $6 \times 10^{8}$ $\mathrm{J}, 3 \times 10^{5} \mathrm{Ns}$, and $5.2 \times 10^{4} \mathrm{Ns}$, respectively.

From these impact parameters the scaling approaches directly yield a predicted Pwave amplitude at InSight's position.

The application of lunar and terrestrial-derived scaling relationships to Mars is wellestablished (e.g. Daubar et al. (2020)). However, it should be noted that both these approaches involve considerable extrapolation in distance to reach the $3452 \mathrm{~km}$ separation from InSight. Extrapolation is required because comparable (i.e., controlled-source, and with the same momentum and energy) impact events have not previously been seismically recorded on the Moon or Earth (or indeed Mars) at distances greater than $\overline{1200 \mathrm{~km}}$.

\subsubsection{Method 2: Wave propagation modeling using estimated moments}

The second approach predicts the amplitudes of the elastodynamic waves recorded at InSight using wave propagation modeling. Because elastodynamic wave propagation is linear, the amplitude at InSight is directly proportional to the magnitude of the source, and calculations can be easily re-scaled for different estimates of source magnitude (which in these cases is a seismic moment) to yield a range of predicted amplitudes.

The seismic moment is thus the primary determinant of peak $\mathrm{P}$-wave amplitude. Several approaches have been proposed to estimate the seismic moment of an impact, with an uncertainty that spans two orders of magnitude (Daubar et al., 2018). Here we derive two independent estimates of the seismic moment: (A) using the seismic moment scaling relation of Teanby and Wookey (2011), and (B) using impact physics modeling codes to simulate the non-linear plastic behaviour and relevant shock physics at the CMBD impact site. 
A) Scaling-based moment estimates Rearranging equations (5) and (6) of Teanby and Wookey (2011) provides an empirically-derived relationship between seismic moment $(M)$ and impact kinetic energy $(E)$, via $M=\left(k_{s} E / 4.8 \times 10^{-9}\right)^{0.81}$, where $k_{s}$ is the seismic efficiency of the impact.

While there remains considerable uncertainty in the most appropriate value for the seismic efficiency of small impacts on Mars (Teanby \& Wookey, 2011; Daubar et al., 2018; Wójcicka et al., 2020), to derive a plausible upper bound on the seismic moment of the CMBD impact we adopt a value of $k_{S}=5 \times 10^{-4}$ (Teanby, 2015; Daubar et al., 2018), which yields a seismic moment $M=1.3 \times 10^{11} \mathrm{Nm}$. This estimate has at least an order of magnitude uncertainty.

B) Impact physics hydrocode simulations To estimate the seismic moment of the CMBD impact in an independent way we use the iSALE2D (Amsden et al., 1980; Collins et al., 2004; Wünnemann et al., 2006) and HOSS (Hybrid Optimization Software Suite; Munjiza, 2004; Lei, Rougier, Knight, \& Munjiza, 2014; Knight et al., 2020) impact physics codes to simulate the impact and wave generation process on millisecond timescales.

Realistic simulations of highly oblique impacts such as the $10^{\circ}$ from horizontal impact of the CMBDs such as the M2020 CMBD impact are extremely challenging. Whilst HOSS is capable of such simulations (iSALE2D is not), these are executable only with lower spatial resolution and over a shorter duration than simulations with vertical impactors.

Therefore, to provide the most robust prediction possible, we simulated both the CMBD collision with the surface as a vertical impact of same $\left(3 \times 10^{5} \mathrm{Ns}\right)$ using both iSALE2D and HOSS (at high resolutions and longer timescales), and as an oblique impact in HOSS (at a lower resolution and shorter timescales). These are labelled as scenario (a) and (b) below.

The trade-off between resolution and duration versus realism means that we cannot claim that one of these cases is 'better' or 'more accurate' than the other. Of the two numerical model estimates, the vertical impact simulation is expected to provide an upper bound on the seismic moment as it maximises the coupling of the impactor's energy with the ground.

In oblique impacts such as this, the horizontal momentum contributes to the crater formation processes and the vertical component alone significantly under-estimates the scalar seismic moment. Because of this, our 2D hydrocode simulations (i.e. those which use a vertical impactor) use the total momentum $\left(3 \times 10^{5} \mathrm{Ns}\right)$ as initial impact momentum.

The scalar seismic moment of the impact was calculated differently for the different simulation approaches. The scalar seismic moment calculated from the iSALE2D simulation results uses a combination of three methods that each provide a measure of either the scalar seismic moment or the diagonal components of the full seismic moment tensor (Wójcicka et al., 2020). The method used to determine the seismic moment from the HOSS simulation provides information about the full seismic moment tensor, including off-diagonal terms. Further details are provided in the Supplement.

\section{Material models [added paragraphs]}

In the iSALE2D simulation, the balance mass was modelled using the Tillotson equation of state (Tillotson, 1962) and the Johnson-Cook strength model (Johnson \& Cook, 1983) with parameters appropriate for tungsten.

To approximate the local geological conditions at Jezero Crater, the target was modelled as a porous basaltic regolith of bulk density $\rho=1589 \mathrm{~kg} / \mathrm{m}^{-3}$ and sound speed $c_{B}=857 \mathrm{~m} / \mathrm{s}$, using the Tillotson equation of state combined with the $\varepsilon$ - $\alpha$ poros- 
ity model (Wünnemann et al., 2006; Collins et al., 2011) and the Lundborg strength model (Lundborg, 1968). Full material model parameters are show the supplementavailable in supplementary material Table S1 or in user-ready format from Wójcicka and Froment (2020).

The HOSS model was configured to be as close to the iSALE2D initial conditions and material models as possible, enabling an accurate but independent method of verifying the derived seismic moment.

In HOSS, the CMBD was modelled using the same equation of state as detailed above for iSALE2D. The HOSS equation of state for the porous target material takes the form of a user-defined curve relating pressure and volumetric strain in a regime of elastic deformation at low stresses, followed by a regime of plasticity and pore-crushing at higher stresses. This model of martian regolith was recently validated based on laboratory hypervelocity impact experiments, conducted in a martian regolith proxy made of loose pumice sand (Richardson \& Kedar, 2013; Froment et al., 2020). In this work, parameters for porosity and sound speed were modified so that the material behaviour replicated, as far as possible, that used in the iSALE2D $\varepsilon-\alpha$ model. A comparison between iSALE2D and HOSS respective parameters can be found in the supplement.

iSALE2D Modeling [added paragraphs]

The shape of the CMBD in iSALE2D is approximated as a tungsten sphere of radius $9.6 \mathrm{~cm}$ and mass $75 \mathrm{~kg}$. The mesh used in the simulations is cylindrically symmetric, approximately $30 \mathrm{~m}$ in radius. The impact-generated shockwave is tracked at high resolution until it decays to a purely linear elastodynamic wave. The arget is a porous basaltic regolith, approximating the local geological conditions at Jezero Crater. Its bulk density is $\rho=1589 \mathrm{~kg} / \mathrm{m}^{-3}$ and-sound speed is $\epsilon_{B}=857 \mathrm{~m} / \mathrm{s}$.

To estimate the seismic moment in the vertical impact case with iSALE2D we follow the three approaches described by Wójcicka et al. (2020). The first approach is based on Müller (1973), which expresses seismic scalar moment, $M_{1}$, in terms of a hemispherical surface surrounding the impact that is moved by an average residual displacement. The second approach is based on Walker (2003) and provides an estimate of the radial component of seismic moment, $M_{r r}$. The final approach was adapted from the Gudkova-Lognonné model (Lognonné et al., 2009) and returns the vertical seismic moment, $\overline{M_{z z}}$, calculated from total momentum transferred to the target during impact. The arithmetic mean of the three seismic moment values was taken to produce a single representative value of the scalar moment, $M_{0}$, to be used in later calculations.

\section{HOSS modeling [added paragraph]}

HOSS uses the Lagrangian description, and is based on the Finite Discrete Element Method (Munjiza, 2004; Lei et al., 2014)). This hybrid representation merges continuum solutions for the calculation of stresses as a function of deformation with the Discrete Element Method for the resolution of fracture, fragmentation, and contact interaction. Impact simulations are conducted in 3D, and unlike iSALE2D need not be cylindrically symmetric.

Two impact geometries are used to simulate a CMBD impact in HOSS:

(a) The first scenario assumes a vertical incidence and a $4000 \mathrm{~m} / \mathrm{s}$ impact velocity. The target geometry is a $30^{\circ}$ cylindrical sector with a height of $27 \mathrm{~m}$. The $3 \mathrm{D}$ mesh is composed of $\sim 533,000$ elements with a minimum size of $1.2 \mathrm{~cm}$ The minimum size of $3 \mathrm{D}$ elements is $1.2 \mathrm{~cm}$.

(b) The second scenario (the 'oblique' case) accounts for the $10^{\circ}$ thefrom horizontal impact angle by modeling the target as thea quarter of a sphere cut $\overline{\text { along }}$ the $x$ - 
$z$ and $x-y$ planes, with radius $12 \mathrm{~m}$. This scenario is conducted at lower spatial and temporal resolutions than scenario (a), with minimum element size of $1.8 \mathrm{~cm}$ - note that this is $50 \%$ larger than in scenario (b).

The total mesh comprises $~ 714,000$ elements with a minimum size of $1.8 \mathrm{~cm}$, and is thus less precise than the vertical case.

The approach used here to compute the seismic moment is different from that of iSALE2D and relies on the notion of Stress Glut developed by Backus and Mulcahy (Backus \& Mulcahy, 1976a, 1976b). This method was applied to planetary impacts in the work of Lognonné et al. (1994) and Gudkova et al. (2015). We derive a second rank seismic moment tensor with six independent components. Here, the effect of material shear strength is accounted for and contributes additional diagonal and non-diagonal terms to the stress glut tensor. The expression of the stress glut, with opposite sign conventions to Lognonné et al. (1994), is the following:

$$
\Pi_{i j}(t)=\Psi_{i j}(t)-S_{i j}(t)+\left(\rho v_{i} v_{j}\right)(t)
$$

where $\Psi_{i j}$ is the the modelled elastic Hooke stress deriving from impact-generated deformation, $S_{i j}$ is the true stress in the material, and $\rho v_{i} v_{j}$ is the Reynolds momentum transport due to crater and ejecta formation. The expression of the timevarying moment tensor in the volume $V$ of the impacted target is then (after Lognonné et al., 1994, eq. 16):

$$
M_{i j}(t)=\int_{V} \Pi_{i j}(t) \mathrm{dV}
$$

From this tensor, a scalar seismic moment $M_{0}=\frac{1}{\sqrt{2}} \sqrt{\sum_{i j} M_{i j}^{2}}$ is derived.

Wave propagation modeling Synthetic waveforms with an isotropic source are generated using Instaseis (van Driel et al., 2015) to retrieve pre-computed Green's function databases prepared for the InSight mission (Ceylan et al., 2017). These are accurate up to a frequency of $1 \mathrm{~Hz}$, and . These are then rescaled using the scalar seismic $\underline{\text { moments; }}$, derived for the CMBD impacts as detailed above.

In this paper, we consider the structural model EH45TcoldCrust1 with attenuation (Rivoldini et al., 2011), which has been used in previous benchmark modeling of impact signals on Mars (Daubar et al., 2018). While modelled waveform amplitudes vary slightly between different structural models, the variations associated with different models are far lower than the uncertainty of the estimated seismic moment of the impact. Given the uncertainties in modeling the focal mechanism for a hypersonic impact (see Daubar et al. (2018) for more details), the use of an isotropic (explosive) source is a standard and justifiable assumption. If this methodology is applied to other contexts where a different source radiation pattern is desired, the extension to using a full second-rank moment tensor in Instaseis is simple.

\section{Results}

\subsection{Acoustic signal}

Fig. 3 presents the trajectories of the spacecraft and CMBD and acoustic ray-tracing simulations. The acoustic energy release at any point in time is dependent on both the velocity of the entry vehicle and the atmospheric density (and hence, the spacecraft altitude). The spacecraft reaches the point of maximum energy releasedissipates the most energy into the atmosphere at the point of maximum aerodynamic deceleration, or approximately $30 \mathrm{~km}$ above the surface and 90 seconds after atmospheric entry interface. 
a)

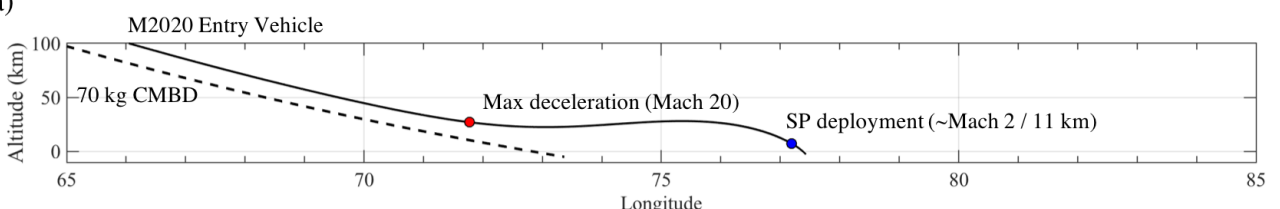

b)

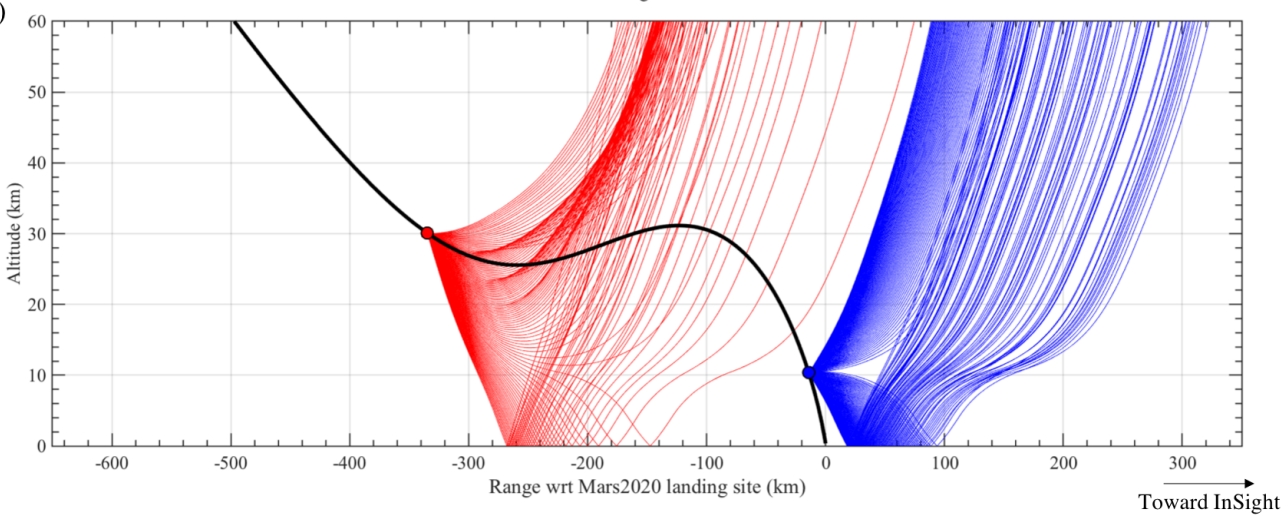

Figure 3. Panel a) shows the entry trajectories of the CMBDs and Mars 2020 entry vehicle (solid and dashed curves, respectively) CMBD separation occurs far off to the top left of the graphic ( $\sim 1450 \mathrm{~km}$ altitude and $\sim 3330 \mathrm{~km}$ downrange). The red dot marks the calculated point of maximum of deceleration (where the emission of acoustic energy into the atmosphere is highest), and the blue dot marks the estimated location of the Supersonic Parachute (SP) opening, after which the spacecraft rapidly becomes subsonic. Panel b) illustrates the infrasound propagation paths on Mars at the time of landing, in red for a source at $30 \mathrm{~km}$ height at the point of maximum deceleration, and in blue for an acoustic source at $11 \mathrm{~km}$ where the SP deployment occurs. 
Acoustic energy emitted at altitudes above $10 \mathrm{~km}$ reflects off the surface back into the atmosphere at too steep an angle to be refracted into the waveguide and propagate toward the lander. Therefore, the acoustic signal produced around the time at which Mars 2020 is undergoing maximum deceleration will not likely be detectable by InSight due to the geometry of the waveguide layer.

Below $10 \mathrm{~km}$, acoustic energy from the decaying shock front may become trapped between the wind layers in the atmosphere and the surface, and hence propagate for long distances. However, the amount of acoustic energy emitted will decrease substantially as the entry vehicle's parachute deploys and it passes into the subsonic regime, around $140 \mathrm{~s}$ prior to landing and approximately $11 \mathrm{~km}$ above the surface. This signal will therefore, with high confidence, not be detectable. A more speculative discussion on this topic which includes a comparison to the APSS noise floor is included such that this methodology can be easily extended to other contexts in Sec. 4.3.3.

The impact of the CMBDs with the ground will generate an substantial acoustic signal which will propagate up into the atmosphere. Due to the complexities of this signal's generation and propagation, it is not currently possible to meaningfully estimate its amplitude at InSight's position. Again, for a more speculative discussion, see Sec 4.3.3.

\subsection{Seismoacoustic coupled signal}

Acoustic ray tracing predicts a sonic boom swath (a 'carpet' in which waves reach the surface directly, i.e. without bouncing off of it first) of width no more than $100 \mathrm{~km}$. We estimate a maximum surface overpressure in this region of $0.9 \mathrm{~Pa}$ with a fundamental frequency of $0.5 \mathrm{~Hz}$, which is attributable to the portion of the sonic boom generated at $25 \mathrm{~km}$ height. At this position, the spacecraft is travelling fast enough to still generate a substantial shockwave (Mach 15).

Using our calculated air-to-ground coupling factor of $4 \times 10^{-6} \mathrm{~ms}^{-1} \mathrm{~Pa}^{-1}$, the 0.9 $\mathrm{Pa}$ overpressure translates into a ground deformation velocity of $3.6 \times 10^{-6} \mathrm{~ms}^{-1}$ at the landing site.

Modelling a seismic source of this magnitude using Instaseis suggests a maximum P-wave amplitude no larger than $2 \times 10^{-11} \mathrm{~m} / \mathrm{s}$ at InSight's location. The average noise spectrum is discussed below in Sec. 4.2, but in short this is substantially below the noise floor and hence will not be detectable.

\subsection{Elastodynamic seismic signal}

As per Sec. 2.3.2, the use of two independent methods (scaling laws and shock physics simulations) to estimate the amplitude of the elastodynamic seismic signal at InSight's position yields a spread of values for the seismic deformation velocity below the lander. From a detectability perspective, the highest of these values corresponds to the 'reasonable best case' scenario, as discussed in Sec. 4).

\subsubsection{Method 1: Empirical scaling relationships}

Figure 4 presents estimates of the peak $\mathrm{P}$-wave amplitude as a function of downrange distance for the CMBD impact, as compared to data from artificial lunar (Latham, Ewing, et al., 1970) and terrestrial missile impact experiments (Latham, McDonald, \& Moore, 1970). These form the basis of our empirical scaling estimates.

The vertical offset between each scaling line occurs because of the different approaches used to scale the results of the experimental data to the Mars 2020 CMBD impact scenario (see Sec. 2.3.1 or Wójcicka et al. (2020) for more details): 


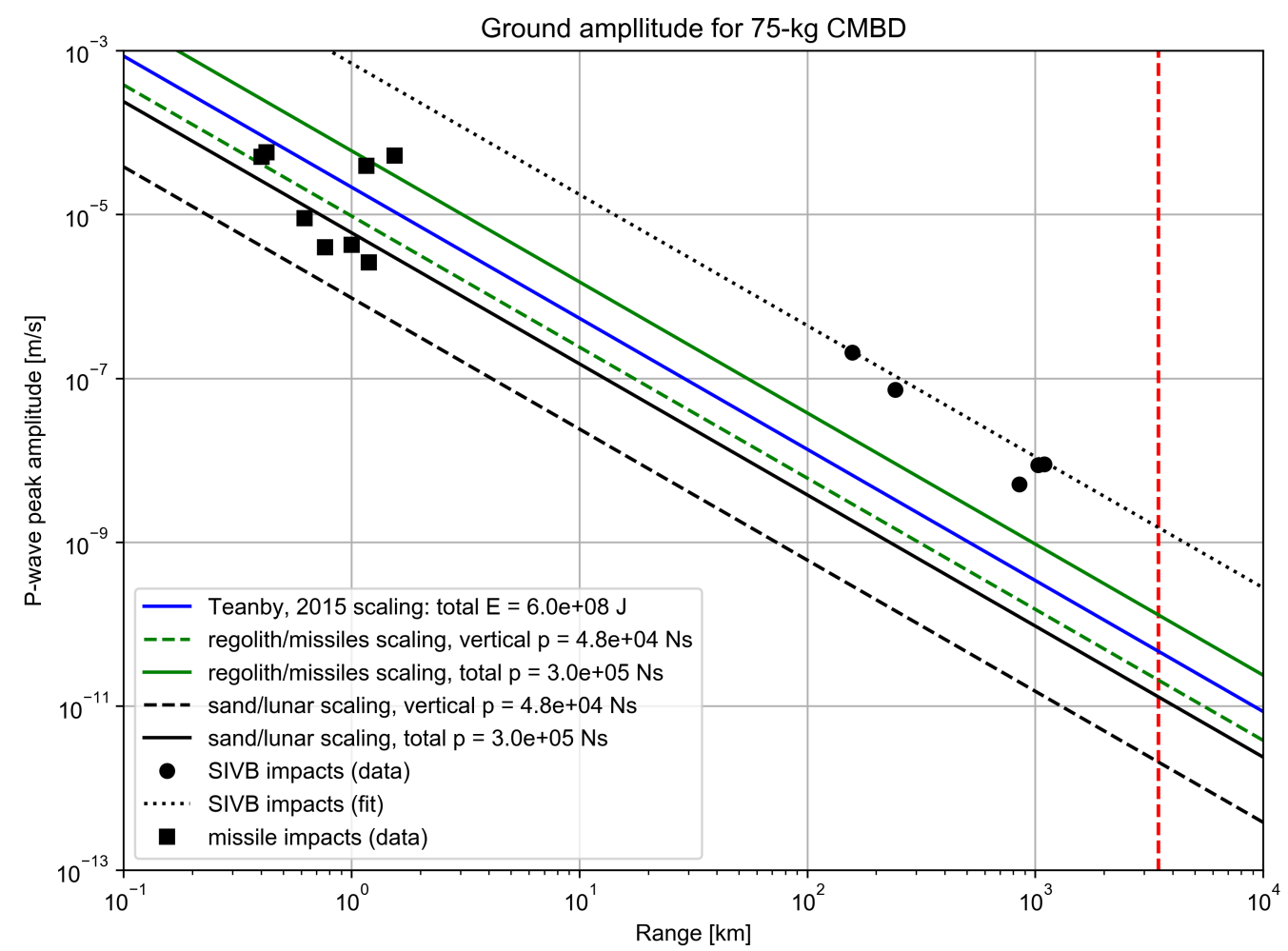

Figure 4. [Added figure] P-wave peak amplitude versus range estimates for the CMBD impact based on different scaling approaches discussed in Section 3.3.1. The solid blue line shows the estimate based on scaling P-wave peak amplitude by the square-root of the impact energy as described by Teanby (2015). The other lines show estimates based on scaling P-wave peak amplitude for the missile data (green lines) or lunar impact data (black lines), in each case with two lines corresponding to scaling by the total impactor momentum (solid lines) or vertical impactor momentum (dashed lines). The dotted black line is a fit to the lunar data, which as discussed below is distant in parameter space from the CMBD impacts and hence is significantly separated from the other scaling lines. The red vertical line marks the distance of InSight from the estimated CMBD impact point. 
- The solid blue line shows scaling by the square root of impact energy.

- The green lines are based on terrestrial missile data, scaled by total (solid) and vertical momentum (dashed).

- The black lines are based on the lunar impact data scaled by total (solid) and vertical (dashed) momentum. The dotted black line is a fit to the data.

The Mars 2020 CMBD impactor momentum and kinetic energy of $3 \times 10^{5} \mathrm{Ns}$ and $6 \times 10^{8} \mathrm{~J}$ are of similar magnitude to the terrestrial missile impacts (Latham, McDonald, \& Moore, 1970). Hence, the P-wave amplitude estimates based on extrapolation of these data (green and blue lines in Fig. 4) are comparable to the missile data (i.e. the scaling lines pass through the region of the datapoints).

The impact momentum, vertical impact momentum and kinetic energy of the lunar impacts (black lines), on the other hand, are approximately 120, 640 and 75 times larger than their corresponding values for the CMBD impact, respectively. As such, a sizable extrapolation in energy or momentum must be performed, in order to use the lunar data to make predictions of the peak P-wave amplitudes for the CMBD impact being considered here.

These differing approaches result in a large range in estimated P-wave peak amplitude at InSight when the trend line based on the lunar experimental data (dotted line) is re-scaled to the CMBD impact by momentum, vertical momentum or the square root of the kinetic energy (blue and black lines).

The lower and upper bounds on the P-wave amplitudes at InSight's position from the different methods are:

- $2.1 \times 10^{-12}$ and $1.3 \times 10^{-11} \mathrm{~m} / \mathrm{s}$ from lunar-based impact momentum scaling

- $2.1 \times 10^{-11}$ and $1.3 \times 10^{-10} \mathrm{~m} / \mathrm{s}$ from terrestrial-based missile scaling

- $5_{-3.5}^{+10} \times 10^{-11} \mathrm{~ms}^{-1}$ from the Teanby (2015) scaling

Note that in the first two cases the lower and upper bounds come from using the vertical and total momentum, respectively; whilst in the latter case the uncertainty is experimentally derived and hence differently presented.

The resulting overall range of peak $\mathrm{P}$-wave velocities at the distance of InSight using these three methods is is between $2.1 \times 10^{-12}$ and $1.3 \times 10^{-10} \mathrm{~ms}^{-1}$ These results are plotted and compared to other derived values for the purposes of estimating detectability in Fig. 6.

\subsubsection{Method 2: Wave propagation modeling with an estimated seismic moment}

A) Scaling based moment estimate While there remains considerable uncertainty in the most appropriate value for the seismic efficiency of small impacts on Mars (Teanby \& Wookey, 2011; Daubar et al., 2018; Wójcicka et al., 2020), to derive a plausible upper bound on the seismic moment of the CMBD impact we adopt a value of $k_{s}=5 \times 10^{-4}$ (Teanby, 2015; Daubar et al., 2018), which yields a seismic moment $M=1.3 \times 10^{11} \mathrm{Nm}$. This estimate has at least an order of magnitude uncertainty.

B) Impact physics hydrocode simulations In the case where the CMBD impact of one CMBD is approximated as a vertical impact scenario (a) from Sec. 2.3.2), iSALE2D predicts a scalar seismic moment of $5.85 \pm 1.5 \times 10^{8} \mathrm{Nm}$ whilst HOSS predicts a moment of $1.79 \times 10^{9} 2.97 \times 10^{9} \mathrm{Nm}$. The factor-of-threefive discrepancy between these two values is likely due to differences in the way that the ejecta from the CMBD crater is modelled and in how the surface material is parameterised. As described in the 
supplementary materialsection 2.3 .2 , each moment estimate wasmust be computed using a different mathematical approach due to the simulation methods used, which will also introduce discrepancyies.

In the case of a highly oblique CMBD impact (scenario (b) from Sec. 2.3.2), the HOSS simulation results yield a scalar seismic moment of $0.76 \times 10^{9} \mathrm{Nm} 0.92 \times 10^{9} \mathrm{Nm}$, which is within the range of estimates of the scalar moment of the vertical impact approximation. We note, however, that iIn this case, the scalar seismic moment is dominated by onepresents a significant off-diagonal component of the moment tensor (shear in the vertical and along-trajectory directions), whereas the diagonal terms of the moment tensor dominate in the vertical impact case (Table 1). This suggests that the use of an isotropic moment tensor source approximation in our wave propagation modeling to represent a highly oblique impact source may introduce an additional uncertainty in P-wave amplitude that should be explored in further work but is beyond the scope of this paper.

The eombinedarithmetic mean of the estimates of scalar seismic moment suggests

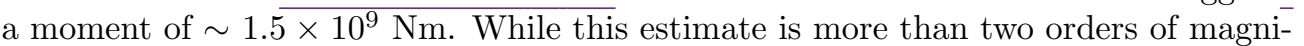
tude less than the estimate of $1.3 \times 10^{11} \mathrm{Nm}$ based on the impact energy-moment scaling relationship of Teanby and Wookey (2011) (using an assumed $k_{s}$ of $5 \times 10^{-4}$, as described in Section 2.3.2 A), it is consistent with other estimates of seismic moment (in both value and difference from other estimates) for impacts of similar momentum in terrestrial, lunar, and martian contexts (Gudkova et al., 2015; Daubar et al., 2018; Wójcicka et al., 2020). Possible reasons for this disparity are discussed in Sec. 4.2.

We therefore consider a predicted range for the seismic moment of $1.0 \times 10^{9}-1.3 \times 10^{11} \mathrm{Nm} 1.5 \times 10^{9}$ $1.3 \times 10^{11} \mathrm{Nm}$, which we are confident bounds the 'true' seismic moment. This can then be used to scale, for scaling the results of our wave propagation modeling.

Using these limits on the source moment to linearly re-scale seismogram velocity amplitudes, as discussed in Sec 2.3.2, yields amplitudes in the range $2.0 \times 10^{-14} \mathrm{~ms}^{-1}$ (corresponding to the lower bound of $1.5 \times 10^{9} \mathrm{Nm}$ ) and $2.0 \times 10^{-12} \mathrm{~ms}^{-1}$ (corresponding to the upper bound predicted moment of $1.3 \times 10^{11} \mathrm{Nm}$ ).

These upper and lower values $\left(v_{u}\right.$ and $v_{l}$ respectively) bound a predicted range of amplitudesground deformation velocities; note that these estimates are entirely independent of the scaling estimates presented in 3.3.1. Seismograms, showing these amplitudes as well as approximate arrival times, are shown in the supplementary material, Fig. S2Fig. 5 .

Possible reasons for the differences between the estimates produced-by the direct scaling relationships and those produced using an intermediate wave propagation stepthe different methods are discussed below.

\section{Discussion}

\subsection{Noise conditions}

The upper range of the amplitude predictions of the elastodynamic seismic wave generated by the CMBD impact with the ground exceeds the noise floor for InSight's SEIS instruments at certain times of day. We now consider how likely this signal is to exceed a signal-to-noise ratio of 1.5 (a reasonable threshold for detection, based on InSight detections of tectonic events) at the predicted time of Perseverance's landing.

Given the highly repeatable meteorological patterns on Mars in the absence of a global dust storm, we estimate the likely noise levels at the time of Perseverance's landing (the local evening of February 18, 2021) using data averaged across twenty evenings from the same period the previous martian year (687 \pm 10 Earth days previously, UTC Earth dates 2019/04/01 to 2019/04/20). 


\begin{tabular}{|c|c|c|}
\hline Moment Component (HOSS results) & Case $(a)$, vertical & Case (b), oblique \\
\hline$M_{x x}[\mathrm{Nm}]$ & $(2.96 \pm 0.60) \times 10^{9}$ & $(3.22 \pm 1.84) \times 10^{8}$ \\
\hline$M_{y y}[\mathrm{Nm}]$ & $(2.96 \pm 0.60) \times 10^{9}$ & $(6.54 \pm 0.12) \times 10^{8}$ \\
\hline$M_{z z}[\mathrm{Nm}]$ & $(0.27 \pm 1.20) \times 10^{9}$ & $(6.30 \pm 1.91) \times 10^{8}$ \\
\hline$M_{x y}[\mathrm{Nm}]$ & 0 & 0 \\
\hline$M_{y z}[\mathrm{Nm}]$ & 0 & 0 \\
\hline$M_{x z}[\mathrm{Nm}]$ & 0 & $(-6.21 \pm 0.1) \times 10^{8}$ \\
\hline Scalar Moment $\mathrm{M}_{0}[\mathrm{Nm}]$ & $(2.97 \pm 0.30) \times 10^{9}$ & $(9.22 \pm 0.30) \times 10^{8}$ \\
\hline Moment Component (iSALE2D results) & \multicolumn{2}{|c|}{ Value } \\
\hline Radial seismic moment, $M_{r r}[\mathrm{Nm}]$ & \multicolumn{2}{|c|}{$4.2 \times 10^{8}$} \\
\hline Vertical seismic moment, $M_{z z}[\mathrm{Nm}]$ & \multicolumn{2}{|c|}{$3.9 \times 10^{8}$} \\
\hline Buried explosion moment, $M_{1}[\mathrm{Nm}]$ & \multicolumn{2}{|c|}{$9.6 \times 10^{8}$} \\
\hline Scalar Moment $\mathrm{M}_{0}[\mathrm{Nm}]$ & \multicolumn{2}{|c|}{$(5.85 \pm 1.5) \times 10^{8}$} \\
\hline Teanby and Wookey (2011) $\mathrm{M}_{0}[\mathrm{Nm}]$ & \multicolumn{2}{|c|}{$1.3 \times 10^{11}$} \\
\hline
\end{tabular}

Table 1. [added table] Table showing the peak and final values of each component of the moment tensorSeismic moment of the CMBD impact obtained from the different hydrocode simulations (top two sections) and the (Teanby \& Wookey, 2011) method (bottom section). The top part of the table shows each moment tensor's components and the scalar seismic moment $M_{0}$ associated with impact scenarios (a) and (b) simulated with HOSS. The total scalar moment $M_{0}$ and moment magnitude $M_{w}$ associated with each scenario are computed using these peak and final values. Results from iSALE2D simulations of the $75 \mathrm{~kg}$ CMBD, calculated using three methods described abovein Section 2.3.2 B, are shown in the bottommiddle part of the table. 

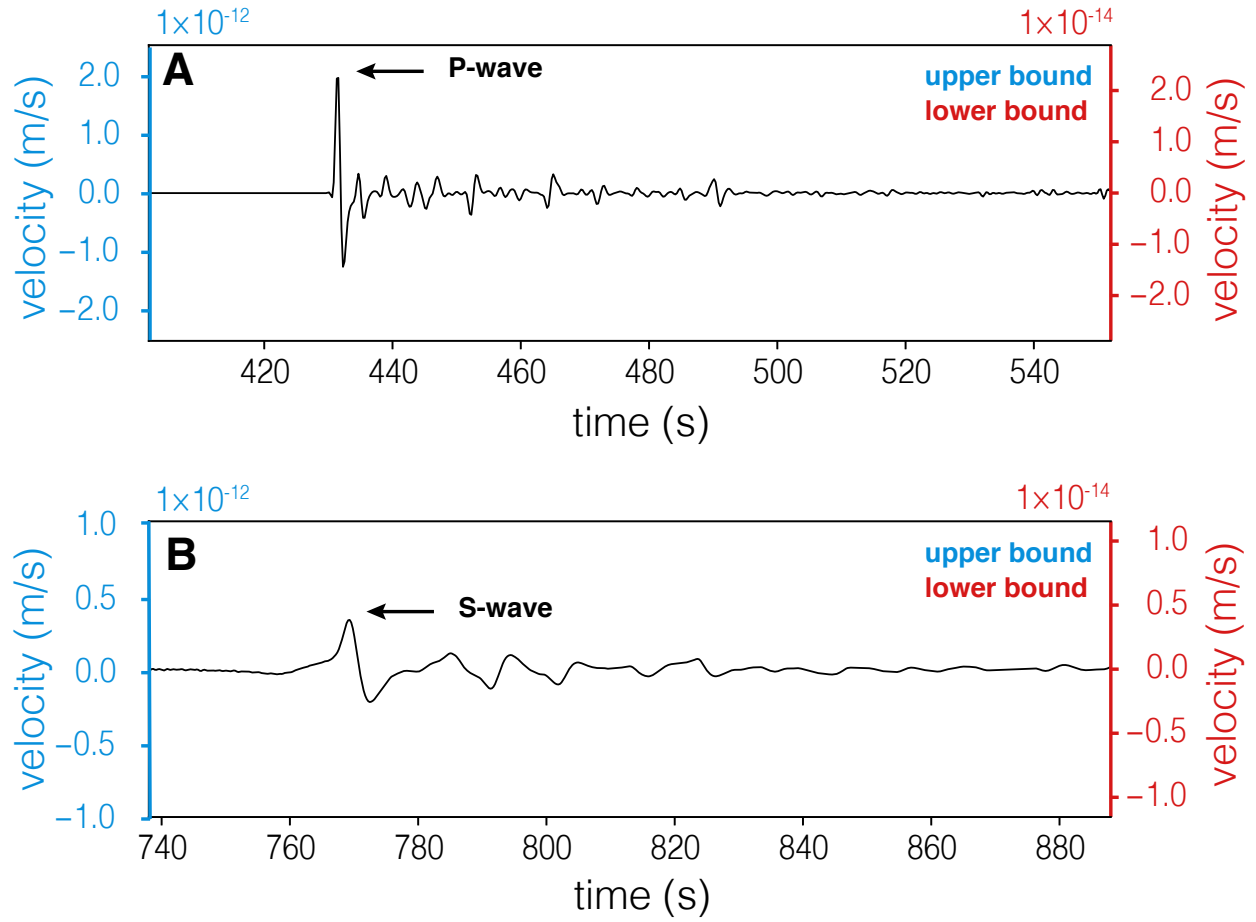

Figure 5. [added figure.] Vertical component Instaseis synthetics calculated for an isotropic moment tensor representation of the CMBD impact. Panels (A) and (B) show close ups of the Pand S-waves, respectively. The vertical scale shown in blue corresponds to velocities calculated assuming a scalar moment $\mathrm{M}_{0}=1.3 \times 10^{11} \mathrm{Nm}$ (the upper bound of moment estimates), and the vertical scale shown in red corresponds to velocities calculated assuming a scalar moment $\mathrm{M}_{0}=$ $1.0 \times 10^{9} \mathrm{NmM}_{0}=1.5 \times 10^{9} \mathrm{Nm}$ (the lower bound of moment estimates). 


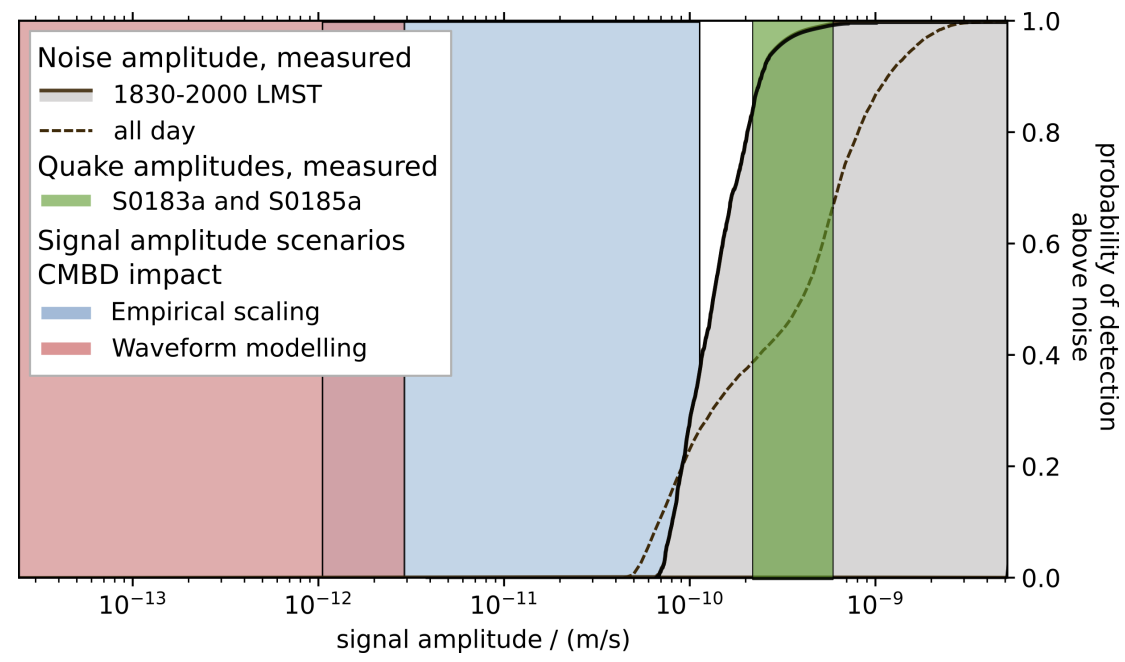

Figure 6. Detection probabilities for seismic signals of certain velocity amplitudes between 0.2 and $0.9 \mathrm{~Hz}$. The solid black curve indicates the noise distribution considering the average signal amplitudes in only the early evening over 20 Sols during the same martian season in 2019, whilst the dashed black curve is for the whole period of 20 Sols. The shaded gray area indicates the regions in which signals are detectable. The blue and red bars mark the P-wave amplitude estimates of the $75-\mathrm{kg}$ CMBD impact, using the empirical scaling and wave propagation modeling estimates, respectively, described earlier in this paper. Vertical lines bounding the different sectors correspond to the upper and lower bounds derived from these methods, for the blue and red sectors respectively (as an example, $v_{u}$ and $v_{l}$ are the vertical edges of the red sector). For comparison, the amplitudes of two previously detected tectonic marsquakes, S0183a and S0185a, located at comparable distances, are plotted in green.

In 2019, these spring evenings (18:30-20:00 LMST at InSight) on Mars were characterised by very low noise levels in the early evening post-sunset within the main seismic band used by the lander $(0.2-0.9 \mathrm{~Hz})$. To account for the temporal variability in the noise levels within this time, we consider the 'probability' of detection as being the fraction of time within the expected arrival window during which a signal of a given amplitude would be at least 1.5 times greater than the noise floor. For reference, we also plot the noise levels for the whole martian day (Sol) in Fig. 6; demonstrating that the noise is on average significantly lower during the evening.

\subsection{CMBD impact: Detection probabilities}

The upper end of the peak amplitude estimates, derived from empirical impact scaling laws (Fig. 6), predicts an amplitude which exceeds the average early evening noise levels by a factor of 1.5 approximately $40 \%$ of the time. This implies that the elastodynamic signal propagating in the ground and induced by the CMBD impact may be detectable at InSight. However, the range of predicted peak ground velocities is substantial. This is not dissimilar to other amplitude predictions for martian impacts (Daubar et al., 2020). This wide range of predicted values is directly attributable to:

- Significant uncertainty in the efficiency of seismic wave generation of oblique impacts, especially in the relationship between impactor momentum and released seismic moment or between impact energy and seismic energy. This is partially a consequence of no impacts having been seismically detected on Mars to date. 
- A lack of prior examples of hypersonic impacts detected at distances greater than $1200 \mathrm{~km}$ on any body, making calibrating scaling relationships challenging. Different approaches to extrapolating these, coupled with differences in material properties between terrestrial soils, lunar regolith and the martian surface, yield estimates that differ by two orders of magnitude depending on the choices made.

- The frequency bands used in estimating scaling relationships are not identical to those used in waveform modeling and predicted noise levels. This is an unavoidable consequence of the frequency content of the available impact data, which are observed at ranges less than $1200 \mathrm{~km}$, so have a somewhat higher frequency content at the receiver location than we expect for the CMBD impacts. For example, the lunar impacts have dominant frequencies of $\sim 2 \mathrm{~Hz}$, whereas we expect the optimal detection band with the lowest noise is $0.2-0.9 \mathrm{~Hz}$ and waveform modeling is performed up to $1 \mathrm{~Hz}$ due to computational limitations.

As the range in estimated peak amplitudes stems from a fundamental lack of observed data in comparable contexts against which to check predictions and understanding of the relevant processes, the range of estimates described here cannot be constrained through further modeling; unless more observational data or more advanced modelling techniques become available. Rather, the uncertainties in our estimates reflect the general lack of knowledge of the excitation and propagation over large distances of impact-generated seismic waves.

Hence, even a single instance of impact detection from a source of known spatial and temporal localisation would therefore be of enormous value. It would offer the potential to better understand impact processes (especially seismic efficiency), enable us to make headway in understanding the sub-surface geology at the landing site (through placing constraints on its seismic properties), as well as offering constraints on the attenuation and average propagation speed along the source-receiver path.

This strengthens the case for listening closely with InSight's instruments for the EDL sequence of Mars 2020. As the upper end of our certainly wide-ranging estimates suggests a reasonable probability of a signal being detected, a positive detection would go a long way tobe extremely useful in resolving the present uncertainty surrounding the propagation of the elastodynamic waves generated by impacts. The enormous advantage that this event holds in attempting to isolate its signal from the noise is that we know exactly the time and location at which it will be produced, and can reasonably estimate when these signals will reach InSight. A non-detection would similarly enable us to further constrain the seismic detectability of impacts on Mars (in effect adding a datapoint on Fig. 4 at the level of the noise floor which represents an upper bound for the seismic signal amplitude), though admittedly by a smaller margin than a positive detection would.

\subsection{Extensions to this work}

Having already discussed some of the limitations encountered in modelling of the CMBD impact signal which future work may seek to address (e.g. high-resolution simulations of oblique impacts and a better characterisation of the equivalent moment tensor), we briefly detail improvements to the modelling which may be made.

It is important to emphasise that we do not expect any of the effects not included in this paper to affect its conclusions, but these are discussed for completeness. They may well be more relevant to other applications of this methodology, for example if an EDL event occurs with receivers in close proximity to the source, or applications to other planetary bodies. 


\subsubsection{Atmospheric attenuation}

Both shock and linear acoustic waves experience an increased attenuation at height, impeding their long-range propagation. The 'classical' (terrestrial) acoustic attenuation due to viscosity, heat conduction, and diffusion is augmented on Mars by the strong molecular relaxation attenuation of $\mathrm{CO}_{2}$ molecules (Williams, 2001). This acts as a low-pass filter, limiting the range of infrasound frequencies which propagate in Mars' atmosphere to between $0.05 \mathrm{~Hz}$ and the order of $\sim 1 \mathrm{~Hz}$ (Martire et al., 2020). The lower bound is the atmospheric cut-off frequency related to the pressure scale height $(2.4 \mathrm{~km})$, whilst the upper bound is related to the high (at least $2 \mathrm{db} / 100 \mathrm{~km}$ ) molecular relaxation from $\mathrm{CO}_{2}$. Further work is needed to more exactly constrain the attenuation dynamics in Mars' atmosphere (Petculescu \& Lueptow, 2007).

-In this study we considered two potential classes of atmospheric signal: the long-distance propagation of acoustic waves in a waveguide (which we concluded do not reach InSight because of the geometry of the EDL and atmospheric structure), and the local-scale propagation of atmospheric-side portion of the coupled seismoacoustic signal in the region known as the sonic boom 'carpet'.

Because the latter is a local-scale effect, we neglected the effects of acoustic attenuation in the atmosphere (and as the predicted amplitude of the seismoacoustic signal at InSight is already below the noise floor, including it would not change our conclusions). However, in other applications of this methodology, it may become important to consider the attenuation of the waves in the atmosphere between the spacecraft and the ground.

\subsubsection{Directionality}

Our acoustic calculations are first-order and do not account for the direction of travel of the spacecraft (the fact that it is travelling almost exactly toward InSight upon arrival). The directionality is likely to have two effects: firstly, the amount of energy directed toward the lander may be reduced (as the majority of the energy is radiated in a direction perpendicular to the vehicle's trajectory). Secondly, a small Doppler shift in the acoustic signal may be apparent. This will increase the frequency of the signal, and it will be more rapidly attenuated as a result.

Both of the consequences of the spacecraft's direction of travel being toward InSight are therefore to reduce the amplitude of any acoustic signal. As the signal is already well below the noise floor at the lander's location, neglecting these effects will not change the conclusions of this paper.

\subsubsection{Low altitude acoustic sources}

As discussed in Sec 3.2, there are two potential low-altitude (i.e. within the tenuous tropospheric waveguide) acoustic sources which occur as a result of the EDL sequence: the CMBD impact with the ground, and acoustic signal produced by the spacecraft once it is sub-sonic. The travel time to InSight for any such signal would be approximately 4-5 hours, though as discussed no detection is expected and this section is included to illustrate a methodology only.

Both of these will produce signals much weaker than the supersonic deceleration of the spacecraft, and in the case of the CMBD impacts quantitatively estimating the acoustic overpressure this will produce is challenging due to the multi-phase and non-linear nature of the problem.

For purely illustrative purposes, we consider an exemplar source of $1 \mathrm{~Pa}$ and $1 \mathrm{~Hz}$ frequency in the Mars 2020 landing region. This is a pressure perturbation $\frac{\Delta P}{P}$ of 
approximately $0.15 \%$. This is substantially larger than most acoustic sources on Mars (Martire et al., 2020) and stronger and lower frequency than we expect either signal to be.

-Even neglecting attenuation, the amplitude of this perturbation after propagating to the distance of InSight is no larger than $3 \times 10^{-4} \mathrm{~Pa}$ (using a $r^{-1}$ scaling) or $5 \times 10^{-6} \mathrm{~Pa}$ (using a $r^{-1.5}$ scaling). For comparison, both values are far below the pressure noise floor of the APSS instrument $\left(\sim 2 \times 10^{-3} \mathrm{~Pa}\right.$ in the 0.1 to $1 \mathrm{~Hz}$ range $)$ (Banfield et al., 2019; Martire et al., 2020).

\subsubsection{Other relevant effects}

For completeness, we also briefly detail other (lower-order) effects which may be relevant in applications of this methodology to other contexts.

The acoustic signal will be affected by surface topography and the temporal evolution of the atmosphere. If an acoustic signal were detected (which we do not expect to be the case here), more detailed consider of the non-linear infrasound propagation in the near-source region should be conducted if the signal is to be clearly associated with an EDL event. It is possible that other kinds of atmospheric waves (e.g. internal gravity waves) may also be excited by an EDL event, though again this is not relevant to the case discussed in this paper.

Modelling of the elastodynamic signal in the solid ground may also include consideration of the source mechanism (as discussed in Sec 3.3.2), and of three-dimensional heterogeneous effects including scattering and local geology.

\section{Conclusions}

We identified three possible sourcestypes of seismoacoustic signals generated by the EDL sequence of the Perseverance landerrover: (1) the propagation of acoustic waves in the atmosphere formed by the decay of the Mach shock, (2) the seismoacoustic air-toground coupling of these waves inducing signals in the solid ground, and (3) the elastodynamic seismic waves propagating in the ground from the hypersonic impacts of the CMBDs.

(1) In the first case (atmospheric propagation), the stratification and wind structure in the atmosphere are such that the strongest signals produced will likely not be detectable at InSight, as they are reflected off the ground back up into the atmosphere. Signals produced in the lower $10 \mathrm{~km}$ of the atmosphere may be trapped and propagate for long distances, however the spacecraft will be subsonic by this point and will not be emitting substantial amounts of acoustic energy into the atmosphere. The Mach shock generated higher in the atmosphere will also have largely dissipated by the time it propagates down to this level. As such no detectable signal is expected. The effects of attenuation and directionality were not included in this model, however as both will serve only to reduce the amplitude of any signal at InSight, they are not considered further in this paper.

(2) In the second case (air-to-ground transmission), the coupling is expected to be very weak. Combined with the substantial distance to InSight, we predict a maximum ground velocity amplitude at SEIS's position of $2 \times 10^{-11} \mathrm{~ms}^{-1}$. This is well below the noise floor at all times of day and hence is not predicted to be detectable.

(3) The generation of seismic waves by an impact comparable to the CMBD impact and the detectability of the seismic signal at large distance are not well understood. 
Using a combination of scaling relationships and wave generation/wave propagation methods, we estimate that the direct body wave arrivals from the impact may be detectable at InSight. In the realistic best-case (and assuming identical weather and noise spectra to the same period one martian year earlier), the requisite signal-to-noise ratio would be sufficient for a positive detection $40 \%$ of the time. It should be noted that our modelling was for only one of the two CMBD impacts. Based on data from the Mars Science Laboratory (Curiosity) landing in 2012, the two CMBDs will impact around $0.1 \mathrm{~s}$ and no more than $1 \mathrm{~km}$ apart. This separation is large enough that craters will not overlap spatially, and any interaction between the two signals will be in the linear propagation regime. As a result, the impact of two rather than one CMBD is unlikely to make a substantial difference to the observed signal, at best increasing the amplitude at InSight by a factor of twowill increase the signal amplitude at InSight by no more than a factor of two, which is less than the uncertainty on the scaling estimates, as described above.

Such a P-wave signal would present itself as a sharp peak in the ground velocity recorded by InSight's SEIS instrument (Fig. 5) approximately $430 \mathrm{~s}$ after the impact of the CMBDs with the ground, just after 15:00 LMST (Perseverance time) or 20:30 LMST (InSight time). This is during the most seismically quiet part of the day at InSight (Banfield et al., 2020; Clinton et al., 2021). If detectable, the S-wave signal would be expected someapproximately $300 \mathrm{~s}$ later; and the travel-time difference would be of use in identifying the signal.

This is likely to be the only impact event with known source parameters during the lifetime of the InSight mission. The Chinese Tianwen-1 is also expected to land on Mars in the spring of 2021 (Wan et al., 2020), but due to a lack of published information on the EDL sequence and hardware, and the time and precise location of its landing, making predictions about the detectability of this signal is not possible; though we eagerly seek clarifying information.

As such, the case for listening for the Mars 2020 signal with InSight's instruments (SEIS and APSS) at the highest possible sampling rates is clear. Whilst this is the first time that such an event detection has been attempted on another planet, InSight's potential on this topic has already proved a source of inspiration in the popular media (Away, Season 1, Episode 8, 2020).

Beyond Mars 2020 and Tianwen-1, this methodology may be extended to future missions including ExoMars (scheduled launch 2022) or Starship.

\section{Acknowledgments}

The InSight Impacts team is grateful to Richard Otero, Erisa Stilley, and Ian Clark of the Jet Propulsion Laboratory for their assistance in modeling and understanding the EDL process. The team also thanks Raphaël Garcia of the Institut Supérieur de l'Aéronautique et de l'Espace for early discussions. BF and TNM are supported by the Natural Environment Research Council under the Oxford Environmental Research Doctoral Training Partnership, and the UK Space Agency Aurora grant ST/S001379/1. Computational resources were supplied in part by TNM's NERC/EPSRC UK National Supercomputer (ARCHER) grant. NW and GSC's research is funded by the UK Space Agency (Grants ST/S001514/1 and ST/T002026/1). SCS acknowledges support from ETH Zürich through the ETH+ funding scheme (ETH+02 19-1: "Planet Mars"). NAT is funded by UK Space Agency Grants ST/R002096/1 and ST/T002972/1. MF and CL's research is funded by the Center of Space and Earth Science of Los Alamos National Laboratory. This research used resources provided by the Los Alamos National Laboratory Institutional Computing Program, which is supported by the U.S. Department of Energy National Nuclear Security Administration under Contract No. 89233218CNA000001. PL, TK, AS, LR and MF acknowledge the support of CNES and of ANR (MAGIS, ANR-19-CE31-0008-08) for SEIS science support. IJD is supported by NASA InSight Participating Scientist grant 
80NM0018F0612. OK acknowledges the support of the Belgian Science Policy Office (BELSPO) through the ESA/PRODEX Program. EKS is supported by the Australian Research Council as part of the Australian Discovery Project scheme (DP170102529). This paper constitutes InSight Contribution Number 191 and LA-UR-20-29568.

Seismograms displayed in the supplementary materialFig. 5 use the wavefield database method Instaseis (van Driel et al., 2015), which is freely and openly available online: https://instaseis.net and is based on AxiSEM (Nissen-Meyer et al., 2014). Data for reproducing hydrocode simulations is available at Wójcicka and Froment (2020). We gratefully acknowledge the developers of iSALE shock physics code used in wave generation modeling (www.isalecode.de). Details of the WASP code used in simulation of atmospheric acoustic propagation can be found in (Dessa et al., 2005).

\section{References}

Amsden, A., Ruppel, H., \& Hirt, C. (1980). SALE: a simplified ALE computer program for fluid flow at all speeds (Tech. Rep.). Los Alamos, NM (United States): Los Alamos National Laboratory (LANL). doi: 10.2172/5176006

Away, Season 1, Episode 8 (Tech. Rep.). (2020). Netflix.

Backus, G., \& Mulcahy, M. (1976a). Moment tensors and other phenomenological descriptions of seismic sources - I. Continuous displacements. Geophysical Journal International, 46(2), 341-361.

Backus, G., \& Mulcahy, M. (1976b). Moment tensors and other phenomenological descriptions of seismic sources - II. Discontinuous displacements. Geophysical Journal International, 47(2), 301-329.

Banerdt, W. B., Smrekar, S. E., Banfield, D., Giardini, D., Golombek, M., Johnson, C. L., ... Wieczorek, M. (2020). Initial results from the InSight mission on Mars (Vol. 13). doi: 10.1038/s41561-020-0544-y

Banfield, D., Rodriguez-Manfredi, J. A., Russell, C. T., Rowe, K. M., Leneman, D., Lai, H. R., .. Banerdt, W. B. (2019). InSight Auxiliary Payload Sensor Suite (APSS) (Vol. 215). doi: 10.1007/s11214-018-0570-x

Banfield, D., Spiga, A., Newman, C., Forget, F., Lemmon, M., Lorenz, R., .. . Banerdt, W. B. (2020). The atmosphere of Mars as observed by InSight. Nature Geoscience, 13(3), 190-198. doi: 10.1038/s41561-020-0534-0

Bass, H. E., \& Chambers, J. P. (2001). Absorption of sound in the martian atmosphere. The Journal of the Acoustical Society of America, 109(6), 3069-3071. Retrieved from https://doi.org/10.1121/1.1365424 doi: $10.1121 / 1.1365424$

Bierhaus, E., McEwen, A., Wade, D., \& Ivanov, A. (2013, Apr). Lunar and planetary science conference, 2013 ..

Borovička, J., Spurný, P., Brown, P., Wiegert, P., Kalenda, P., Clark, D., \& Shrbený, L. (2013). The trajectory, structure and origin of the chelyabinsk asteroidal impactor. Nature, 503(7475), 235-237. doi: 10.1038/nature12671

Ceylan, S., van Driel, M., Euchner, F., Khan, A., Clinton, J., Krischer, L., .. G Giardini, D. (2017). From Initial Models of Seismicity, Structure and Noise to Synthetic Seismograms for Mars. Space Science Reviews, 1-16. doi: $10.1007 / \mathrm{s} 11214-017-0380-6$

Clinton, J. F., Ceylan, S., van Driel, M., Giardini, D., Stahler, S. C., Böse, M., ... Stott, A. E. (2021, January). The Marsquake catalogue from InSight, sols 0-478. Physics of the Earth and Planetary Interiors, 310.

Collins, G. S., Melosh, H., \& Wünnemann, K. (2011). Improvements to the $\epsilon-\alpha$ porous compaction model for simulating impacts into high-porosity solar system objects. International Journal of Impact Engineering, 38(6), 434-439. doi: 10.1016/j.ijimpeng.2010.10.013 
Collins, G. S., Melosh, H. J., \& Ivanov, B. A. (2004). Modeling damage and deformation in impact simulations. Meteoritics \& Planetary Science, 39(2), 217231. doi: 10.1111/j.1945-5100.2004.tb00337.x

Daubar, I., Lognonné, P., Teanby, N. A., Collins, G. S., Clinton, J., Stähler, S., .. . Banerdt, B. (2020, jul). A New Crater Near InSight: Implications for Seismic Impact Detectability on Mars. Journal of Geophysical Research: Planets, 125 (8). doi: 10.1029/2020JE006382

Daubar, I., Lognonné, P., Teanby, N. A., Miljkovic, K., Stevanović, J., Vaubaillon, J., ... Banerdt, W. B. (2018). Impact-Seismic Investigations of the InSight Mission. Space Science Reviews, 214. doi: 10.1007/s11214-018-0562-x

Daubar, I., McEwen, A., Byrne, S., Kennedy, M., \& Ivanov, B. (2013). The current martian cratering rate. Icarus, 225(1), 506-516. doi: 10.1016/J.ICARUS.2013 .04 .009

de Groot-Hedlin, C. D., \& Hedlin, M. A. (2014). Infrasound detection of the chelyabinsk meteor at the usarray. Earth and Planetary Science Letters, 402, 337-345. Retrieved from https://www.sciencedirect.com/science/ article/pii/S0012821X14000417 (Special issue on USArray science) doi: https://doi.org/10.1016/j.epsl.2014.01.031

de Groot-Hedlin, C. D., Hedlin, M. A. H., Walker, K. T., Drob, D. P., \& Zumberge, M. A. (2008). Evaluation of infrasound signals from the shuttle Atlantis using a large seismic network. The Journal of the Acoustical Society of America, 124(3), 1442. doi: 10.1121/1.2956475

Dessa, J. X., Virieux, J., \& Lambotte, S. (2005). Infrasound modeling in a spherical heterogeneous atmosphere. Geophysical Research Letters, 32(12), 1-5. doi: 10 $.1029 / 2005$ GL022867

Devillepoix, H., Cupák, M., Bland, P., Sansom, E., Towner, M., Howie, R., .. others (2020). A global fireball observatory. Planetary and Space Science, 191, 105036.

Edwards, W. N. (2009). Meteor generated infrasound: Theory and observation. In A. Le Pichon, E. Blanc, \& A. Hauchecorne (Eds.), Infrasound monitoring for atmospheric studies (p. 361 - 414). Springer Netherlands. doi: 10.1007/978-1-4020-9508-5-12

Edwards, W. N., Eaton, D. W., McCausland, P. J., ReVelle, D. O., \& Brown, P. G. (2007). Calibrating infrasonic to seismic coupling using the Stardust sample return capsule shockwave: Implications for seismic observations of meteors. Journal of Geophysical Research: Solid Earth, 112(10), 1-13. doi: 10.1029/2006JB004621

Ens, T., Brown, P., Edwards, W., \& Silber, E. tion by bolides: A global statistical study. and Solar-Terrestrial Physics, 80, 208-229.

(2012). Infrasound producJournal of Atmospheric www.sciencedirect.com/science/article/pii/S1364682612000326 doi: https://doi.org/10.1016/j.jastp.2012.01.018

Froment, M., Rougier, E., Larmat, C., Lei, Z., Euser, B., Kedar, S., .. . Lognonné, P. (2020). Lagrangian-based simulations of hypervelocity impact experiments on Mars regolith proxy. Geophysical Research Letters, $47(13)$, e2020GL087393.

Garcia, R. F., Brissaud, Q., Rolland, L., Martin, R., Komatitsch, D., Spiga, A., ... Banerdt, B. (2017). Finite-Difference Modeling of Acoustic and Gravity Wave Propagation in Mars Atmosphere: Application to Infrasounds Emitted by Meteor Impacts. Space Science Reviews.

Giardini, D., Lognonné, P., Banerdt, W. B., Pike, W. T., Christensen, U., Ceylan, S., .. Yana, C. (2020). The seismicity of Mars. Nature Geoscience, 13 , 205-212. doi: 10.1038/s41561-020-0539-8

Grant, J. A., Golombek, M. P., Wilson, S. A., Farley, K. A., Williford, K. H., \& Chen, A. (2018). The science process for selecting the landing site for 
the 2020 mars rover. $\quad$ Planetary and Space Science, 164, 106 - $126 . \quad$ doi: https://doi.org/10.1016/j.pss.2018.07.001

Gudkova, T., Lognonné, P., Miljković, K., \& Gagnepain-Beyneix, J. (2015). Impact cutoff frequency-momentum scaling law inverted from Apollo seismic data. Earth and Planetary Science Letters, 427, 57-65.

Hilton, D. A., \& Henderson, H. R. (1974). Measurements of sonic boom overpressures from apollo space vehicles. The Journal of the Acoustical Society of America, 56 (2), 323-328. doi: 10.1121/1.1903261

Ishihara, Y., Hiramatsu, Y., Yamamoto, M.-y., Furumoto, M., \& Fujita, K. (2012). Infrasound/seismic observation of the hayabusa reentry: Observations and preliminary results. Earth, Planets and Space, 64(7), 655-660. Retrieved from https://doi.org/10.5047/eps.2012.01.003

Johnson, G. R., \& Cook, W. H. (1983). A constitutive model and data from metals subjected to large strains, high strain rates and high temperatures. Proc. 7th Int. Symp. on Ballistics, The Hague, Netherlands.

Karakostas, F., Rakoto, V., Lognonné, P., Larmat, C., Daubar, I., \& Miljković, K. (2018, Nov 27). Inversion of meteor rayleigh waves on earth and modeling of air coupled rayleigh waves on mars. Space Science Reviews, 214(8), 127. doi: 10.1007/s11214-018-0566-6

Karlgaard, C. D., Kutty, P., Schoenenberger, M., Munk, M. M., Little, A., Kuhl, C. A., \& Shidner, J. (2014). Mars science laboratory entry atmospheric data system trajectory and atmosphere reconstruction. Journal of Spacecraft and Rockets, 51(4), 1029-1047. doi: 10.2514/1.A32770

Knight, E. E., Rougier, E., Lei, Z., Euser, B., Chau, V., Boyce, S. H., ... Froment, M. (2020). HOSS: an implementation of the combined finite-discrete element method. Computational Particle Mechanics, 1-23.

Latham, G., Ewing, M., Dorman, J., Press, F., Toksoz, N., Sutton, G., ... Yates, M. (1970). Seismic data from man-made impacts on the moon. Science, 170(3958), 620-626. doi: 10.1126/science.170.3958.620

Latham, G., McDonald, W. G., \& Moore, H. J. (1970). Missile impacts as sources of seismic energy on the moon. Science, 168(3928), 242-245.

Lei, Z., Rougier, E., Knight, E., \& Munjiza, A. (2014). A framework for grand scale parallelization of the combined finite discrete element method in 2d. Computational Particle Mechanics, 1(3), 307-319.

Le Pichon, A., Antier, K., Cansi, Y., Hernandez, B., Minaya, E., Burgoa, B., ... Vaubaillon, J. (2008). Evidence for a meteoritic origin of the September 15, 2007, Carancas crater. Meteoritics \& Planetary Science, 43(11), 1797-1809. doi: https://doi.org/10.1111/j.1945-5100.2008.tb00644.x

Lognonné, P., Banerdt, W. B., Giardini, D., Pike, W. T., Christensen, U., Laudet, P., ... Wookey, J. (2019). SEIS: Insight's Seismic Experiment for Internal Structure of Mars (Vol. 215). doi: 10.1007/s11214-018-0574-6

Lognonné, P., Karakostas, F., Rolland, L., \& Nishikawa, Y. (2016). Modeling of atmospheric-coupled rayleigh waves on planets with atmosphere: From earth observation to mars and venus perspectives. The Journal of the Acoustical Society of America, 140(2), 1447-1468. doi: 10.1121/1.4960788

Lognonné, P., Le Feuvre, M., Johnson, C. L., \& Weber, R. C. (2009). Moon meteoritic seismic hum: Steady state prediction. Journal of Geophysical Research, 114(E12), E12003. doi: 10.1029/2008JE003294

Lognonné, P., Mosser, B., \& Dahlen, F. (1994). Excitation of jovian seismic waves by the Shoemaker-Levy 9 cometary impact. Icarus, 110(2), 180-195.

Lundborg, N. (1968). Strength of rock-like materials. International Journal of Rock Mechanics and Mining Sciences and, 5(5), 427-454. doi: 10.1016/0148 -9062(68)90046-6

Martire, L., Garcia, R. F., Rolland, L., Spiga, A., Lognonné, P. H., Banfield, D., ... Martin, R. (2020, jun). Martian Infrasound: Numerical Modeling and Analysis 
of InSight's Data. Journal of Geophysical Research: Planets, 125(6), 1-34. doi: 10.1029/2020JE006376

Millour, E., Forget, F., Spiga, A., Navarro, T., Madeleine, J.-B., Montabone, L., ... MCD/GCM development Team (2015). The Mars Climate Database (MCD version 5.2). European Planetary Science Congress 2015, 10, EPSC2015-438.

Montabone, L., Forget, F., Millour, E., Wilson, R. J., Lewis, S. R., Cantor, B., ... Wolff, M. J. (2015). Eight-year climatology of dust optical depth on Mars. Icarus, 251, 65-95. doi: 10.1016/j.icarus.2014.12.034

Müller, G. (1973). Seismic moment and long-period radiation of underground nuclear explosions. Bulletin of the Seismological Society of America, 63(3), 847857.

Munjiza, A. (2004). The combined finite-discrete element method. Wiley.

Nakamura, Y., Latham, G. V., \& Dorman, H. J. (1982). Apollo lunar seismic experiment-final summary. Journal of Geophysical Research: Solid Earth, 87(S01), A117-A123. Retrieved from https://agupubs.onlinelibrary.wiley.com/ doi/abs/10.1029/JB087iS01p0A117 doi: https://doi.org/10.1029/ JB087iS01p0A117

Nissen-Meyer, T., van Driel, M., Stähler, S. C., Hosseini, K., Hempel, S., Auer, L., ... Fournier, A. (2014). Axisem: broadband 3-d seismic wavefields in axisymmetric media. Solid Earth, 5(1), 425-445. Retrieved from https:// se.copernicus.org/articles/5/425/2014/ doi: 10.5194/se-5-425-2014

Nunn, C., Garcia, R. F., Nakamura, Y., Marusiak, A. G., Kawamura, T., Sun, D., ... Zhu, P. (2020). Lunar Seismology: A Data and Instrumentation Review. Space Science Reviews, 216. doi: 10.1007/s11214-020-00709-3

Panning, M. P., Beucler, É., Drilleau, M., Mocquet, A., Lognonné, P., \& Banerdt, W. B. (2015). Verifying single-station seismic approaches using Earth-based data: Preparation for data return from the InSight mission to Mars. Icarus, 248(242), 230-242. doi: 10.1016/j.icarus.2014.10.035

Petculescu, A., \& Lueptow, R. M. (2007, February). Atmospheric acoustics of Titan, Mars, Venus, and Earth. Icarus, 186(2), 413-419.

Qamar, A. (1995, 09). Space Shuttle and Meteroid-Tracking Supersonic Objects in the Atmosphere with Seismographs. Seismological Research Letters, 66(5), 612. Retrieved from https://doi.org/10.1785/gssrl.66.5.6 doi: 10.1785/ gssrl.66.5.6

ReVelle, D. O. (1976). On Meteor-Generated Infrasound. Journal of Geophysical Research, 81(7), 1217-1230. doi: 10.1029/ja081i007p01217

ReVelle, D. O., Edwards, W., \& Sandoval, T. D. (2005). Genesis-an artificial, low velocity "meteor" fall and recovery: September 8, 2004 . Meteoritics 83 Planetary Science, 40(6), 895-916. Retrieved from https://onlinelibrary.wiley .com/doi/abs/10.1111/j.1945-5100.2005.tb00162.x doi: https://doi.org/ 10.1111/j.1945-5100.2005.tb00162.x

ReVelle, D. O., \& Edwards, W. N. (2007). Stardust-an artificial, low-velocity "meteor" fall and recovery: 15 january 2006. Meteoritics \& Planetary Science, 42(2), 271-299. Retrieved from https://onlinelibrary.wiley.com/doi/ abs/10.1111/j.1945-5100.2007.tb00232.x doi: https://doi.org/10.1111/ j.1945-5100.2007.tb00232.x

Richardson, J., \& Kedar, S. (2013). An experimental investigation of the seismic signal produced by hypervelocity impacts. In Lunar and planetary science conference (Vol. 44, p. 2863).

Rivoldini, A., Van Hoolst, T., Verhoeven, O., Mocquet, A., \& Dehant, V. (2011). Geodesy constraints on the interior structure and composition of Mars. Icarus, 213(2), 451-472. doi: 10.1016/j.icarus.2011.03.024

Silber, E. A., Brown, P. G., \& Krzeminski, Z. (2015). Optical observations of meteors generating infrasound: Weak shock theory and validation. Journal of Geophysical Research: Planets, 120(3), 413-428. doi: https://doi.org/10.1002/ 


\section{JE004680}

Sorrells, G. G., McDonald, J. A., Herrin, E., \& Der, Z. A. (1971). Earth Motion Caused by Local Atmospheric-Pressure Changes. Geophysical Journal of the Royal Astronomical Society, 26(1-4), 83-\&.

Spiga, A., Banfield, D., Teanby, N. A., Forget, F., Lucas, A., Kenda, B., ... Banerdt, W. B. (2018). Atmospheric Science with InSight. Space Science Reviews, 214(7). doi: 10.1007/s11214-018-0543-0

Stevanović, J., Teanby, N. A., Wookey, J., Selby, N., Daubar, I. J., Vaubaillon, J., \& Garcia, R. (2017). Bolide Airbursts as a Seismic Source for the 2018 Mars InSight Mission. $\quad$ Space Science Reviews, 211(1-4), 525-545. doi: 10.1007/s11214-016-0327-3

Tancredi, G., Ishitsuka, J., Schultz, P. H., Harris, R. S., Brown, P., Revelle, D. O., ... Dalmau, A. (2009). A meteorite crater on Earth formed on September 15, 2007: The Carancas hypervelocity impact. Meteoritics \& Planetary Science Archives, 44(12), 1967-1984. doi: https://doi.org/10.1111/ j.1945-5100.2009.tb02006.x

Tauzin, B., Debayle, E., Quantin, C., \& Coltice, N. （2013). Seismoacoustic coupling induced by the breakup of the 15 february 2013 chelyabinsk meteor. Geophysical Research Letters, 40(14), 3522-3526. Retrieved from https://agupubs.onlinelibrary.wiley.com/doi/abs/10.1002/grl.50683 doi: https://doi.org/10.1002/grl.50683

Teanby, N. A. (2015). Predicted detection rates of regional-scale meteorite impacts on Mars with the InSight short-period seismometer. Icarus, 256, 49-62.

Teanby, N. A., \& Wookey, J. (2011). Seismic detection of meteorite impacts on Mars. Physics of the Earth and Planetary Interiors, 186, 70-80. doi: 10.1016/ j.pepi.2011.03.004

Tillotson, J. H. (1962). Metallic Equations of State for Hypervelocity Impact. General Atomic.

van Driel, M., Ceylan, S., Clinton, J. F., Giardini, D., Alemany, H., Allam, A., ... Zheng, Y. (2019). Preparing for InSight: Evaluation of the blind test for martian seismicity (Vol. 90) (No. 4). doi: 10.1785/0220180379

van Driel, M., Krischer, L., Stähler, S. C., Hosseini, K., \& Nissen-Meyer, T. (2015). Instaseis: Instant global seismograms based on a broadband waveform database. Solid Earth, 6, 701-717. doi: 10.5194/se-6-701-2015

Varnier, J., Le Pape, M.-C., \& Sourgen, F. (2018). Ballistic wave from projectiles and vehicles of simple geometry. AIAA Journal, 56(7), 2725-2742. doi: 10 $.2514 / 1 . J 056239$

Walker, J. D. (2003). Loading sources for seismological investigation of asteroids and comets. International Journal of Impact Engineering, 29(1-10), 757-769. doi: 10.1016/J.IJIMPENG.2003.10.022

Wan, W. X., Wang, C., Li, C. L., \& Wei, Y. (2020). China's first mission to Mars (Vol. 4) (No. 721). doi: 10.1038/s41550-020-1148-6

Williams, J.-P. (2001, March). Acoustic environment of the Martian surface. Journal of Geophysical Research: Planets, 106(E3), 5033-5041.

Wójcicka, N., Collins, G. S., Bastow, I. D., Teanby, N. A., Miljković, K., Rajšić, A., ... Lognonné, P. (2020). The seismic moment and seismic efficiency of small impacts on Mars. Journal of Geophysical Research: Planets. doi: 10.1029/2020JE006540

Wójcicka, N., \& Froment, M. (2020). nwojcicka/listening-for-landing-SI: listeningfor-landing-SI. Zenodo. Retrieved from https://doi.org/10.5281/zenodo .4291898 doi: $10.5281 /$ zenodo.4291898

Wünnemann, K., Collins, G. S., \& Melosh, H. (2006). A strain-based porosity model for use in hydrocode simulations of impacts and implications for transient crater growth in porous targets. Icarus, $180(2), 514-527$. doi: 10.1016/J.ICARUS.2005.10.013 